\title{
Debonding of confined elastomeric layer using cohesive zone model
}

\author{
Bikramjit Mukherjee $^{a}$, David A. Dillard ${ }^{a, *}$, Robert B. Moore ${ }^{b}$, Romesh C. Batra ${ }^{a}$ \\ a Department of Biomedical Engineering and Mechanics, M/C 0219, Virginia Polytechnic Institute and State University, Blacksburg, VA 24061, USA \\ ${ }^{\mathrm{b}}$ Department of Chemistry, M/C 0212, Virginia Polytechnic Institute and State University, Blacksburg, VA 24061, USA
}

\section{A R T I C L E I N F O}

\section{Article history:}

Accepted 3 October 2015

Available online 12 December 2015

\section{Keywords:}

Fingerlike instability

Wavy or undulatory debond front

Cohesive zone model (CZM)

Elastomeric interlayer

Debonding

\begin{abstract}
A B S T R A C T
Wavy or undulatory debonding is often observed when a confined/sandwiched elastomeric layer is pulled off from a stiff adherend. Here we analyze this debonding phenomenon using a cohesive zone model (CZM). Using stability analysis of linear equations governing plane strain quasi-static deformations of an elastomer, we find (i) a non-dimensional number relating the elastomer layer thickness, $h$, the long term Young's modulus, $E_{\infty}$, of the interlayer material, the peak traction, $T_{c}$, in the CZM bilinear tractionseparation (TS) relation, and the fracture energy, $\mathcal{G}_{c}$, of the interface between the adherend and the elastomer layer, and (ii) the critical value of this number that provides a necessary condition for undulations to occur during debonding. For the elastomer modeled as a linear viscoelastic material with the shear modulus given by a Prony series and a rate-independent bilinear TS relation in the CZM, the stability analysis predicts that a necessary condition for a wavy solution is that $T_{c}{ }^{2} h / \mathcal{G}_{c} E_{\infty}$ exceed 4.15 . This is supported by numerically solving governing equations by the finite element method (FEM). Lastly, we use the FEM to study three-dimensional deformations of the peeling (induced by an edge displacement) of a flexible plate from a thin elastomeric layer perfectly bonded to a rigid substrate. These simulations predict progressive debonding with a fingerlike front for sufficiently confined interlayers when the TS parameters satisfy a constraint similar to that found from the stability analysis of the plane strain problem.
\end{abstract}

(c) 2015 Elsevier Ltd. All rights reserved.

\section{Introduction}

Systems consisting of a soft elastic or viscoelastic layer confined between two stiff substrates occur in numerous industrial applications. One example is manufacturing of bio-implants, which may involve mechanically demolding a soft polymer layer sandwiched between two relatively stiff molds [1]. A frequently observed phenomenon is the occurrence of contact undulations when a stiff layer is separated from the soft layer under tensile tractions. Classical examples include the formation of ripples when a contactor approaches an elastic film bonded to a fixed base [2-4], and wavy debonds in peel [5] and probe tack tests [6-8]. Experimentally, the characteristic spacing, $\lambda$, between two adjacent undulation peaks has been found [2,9] to scale linearly $(\lambda \approx 3-4 h)$ with the thickness $h$ of the confined interlayer while being independent of the interfacial adhesion properties. The linear stability analyses and energy arguments [10-13] have been used to show that undulations result from the competition between the strain energy of the system acting as a stabilizing influence, and the energy associated with the interfacial forces

\footnotetext{
* Corresponding author. Tel.: +1 540231 4714; fax: +1 5402319187.

E-mail address: dillard@vt.edu (D.A. Dillard).
}

(such as van der Waals forces) acting as a destabilizing influence. These approaches give a threshold value of the interaction energy for the onset of instability. For example, it was shown $[10,14]$ that the condition $\frac{A}{6 \pi d^{4}} \geq \frac{2 E}{3 h}$ is necessary for the onset of contact instabilities when a rigid contactor is gradually brought close to an elastic film of thickness $h$ and Young's modulus $E$, where $A$ is the effective Hamaker constant for van der Waals interactions and $d$ the gap between the contactor and the film at the onset of instabilities. Other examples include [15] morphological changes in an elastic film caused by an applied electric field. Combined experimental and linear stability approaches have helped identify a threshold value of the effective voltage as a function of the film stiffness.

As debonding ensues at the interface between an adhesive and an adherend, multiple nonlinear processes such as cavitation and fibrillation may occur at the debonding site. These involve different length scales and contribute to the overall energy dissipation during the creation of the two new surfaces. In a cohesive zone model (CZM) [16,17], the collective influences of these small-scale mechanisms are lumped together into a traction-separation (TS) relation. In this approach the adjoining points on the two sides of an interface are conceived to be connected by a spring of zero length that begins softening with extension (separation) after reaching a critical extension and subsequently breaks upon 


\section{Nomenclature}

Symbols

A Hamaker constant for van der Waals interaction

B Left Cauchy-Green tensor

$d \quad$ Distance between an elastic film and an approaching contactor

$\bar{d} \quad$ Damage variable

$D \quad$ Bending rigidity of the flexible plate

E Young's modulus of the elastomeric interlayer

$h \quad$ Thickness of the elastomeric interlayer

$i \quad=\sqrt{-1}$

$K \quad$ Slope of the rising part of the straight line in the bilinear traction-separation law

$k \quad$ Wavenumber of the $x$ - dependent part of the perturbation to the stream function and the hydrostatic pressure.

$m \quad$ Ratio of the modulus of the spring in the spring-dashpot link to the long-time modulus for the 1-term Prony series

$p \quad$ Hydrostatic pressure not related to strains for incompressible materials

$R \quad$ Reaction force on the rigid adherend

$T \quad$ Normal traction at the interface

$t \quad$ Time

$u, v, w \quad$ Displacement components along $x, y$ and $z$ directions, respectively

$x, y, z \quad$ Axes of the rectangular Cartesian coordinate system when the index $i$ of the system $x_{i}$ has values 1,2 and 3, respectively

Magnitude of the slope of the falling part of the straight line portion of the bilinear traction-separation relation

Applied vertical displacement to the upper adherend

Displacement jump at the interface, also called the contact opening

Dominant wavelength of debonding undulation

Shear modulus of the elastomeric interlayer

Stream function introduced to define displacement components $u$ and $w$

Growth rate of a perturbation

$a_{1}, a_{2} \quad$ Material constants in the constitutive equation used to model finite strain viscoelasticity

$a_{T} \quad$ Shift factor relating the relaxation time at temperature $T$ to that at the reference temperature

$C_{1}, C_{2} \quad$ Constants in the Williams- Landel-Ferry (WLF) equation for $a_{T}$

$E_{\infty}\left(=3 \mu_{\infty}\right)$ Long term Young's modulus ( $=3 \times$ long-term shear modulus) of the viscoelastic material

$E_{0}\left(=3 \mu_{0}\right)$ Instantaneous Young's modulus $(=3 \times$ instantaneous shear modulus $)$ of the viscoelastic material

$F_{t} \quad$ Deformation gradient

$\mathcal{G}_{c} \quad$ Fracture energy of the interface

$g_{R} \quad$ Relaxation function normalized by the instantaneous modulus of the viscoelastic material

$K_{\text {softening }}=T_{c}{ }^{2} / \mathcal{G}_{c}$

$K_{\text {elastic }}=E_{\infty} / h$

$l_{\text {finger }} \quad$ Length of a finger

$p^{n h} \quad$ Non-homogeneous perturbation to the hydrostatic pressure

$T_{c} \quad$ Peak value of the normal traction at the interface

$x_{i} \quad$ Axes of the rectangular Cartesian coordinate system

$\delta_{c} \quad$ Critical displacement jump when damage initiates

$\delta_{f} \quad$ Displacement jump at the initiation of debonding/separation

$\varepsilon_{i j} \quad$ Components of the infinitesimal strain tensor

$\mu_{i} \quad$ Shear modulus of the $i^{\text {th }}$ term in the generalized Maxwell model used to define the relaxation function of the viscoelastic model.

$\mu_{R} \quad$ Relaxation function for the elastomeric interlayer when modeled as a linear viscoelastic material

$\sigma_{i j} \quad$ Components of the stress tensor

$\tau_{i} \quad$ Characteristic relaxation time of the $i^{\text {th }}$ element of the generalized Maxwell model

$\phi_{c 1} \quad$ The lower limit of $\phi$ for debonding instability

$\phi_{c 2} \quad$ The value of $\phi$ beyond which region III sets in

$\psi^{\text {nh }} \quad$ Non-homogeneous perturbation of the stream function

$\tilde{A} \quad$ Area of the interlayer initially bonded to the rigid adherend

$\tilde{p} \quad z$-dependent part of the hydrostatic pressure perturbation

$\tilde{T} \quad$ Temperature

$\tilde{T}_{R E F} \quad$ Reference temperature

$\frac{\partial}{\partial x_{i}} \quad$ Partial derivative (with respect to $x_{i}$ ) operator

$\bar{x} \quad$ Distance along the $x$ axis normalized by the half width of the rigid adherend

$\dot{\Delta} \quad$ Rate of the applied vertical displacement to the upper adherend

$\bar{\delta} \quad$ Contact opening normalized by $\delta_{f}$

$\tilde{\psi} \quad z$-dependent part of the stream function perturbation

$\tilde{\omega} \quad$ The growth rate $\omega$ normalized by $1 / \tau_{1}$ 
reaching a larger limiting value of the extension. In a typical TS relation, it is assumed that the energy associated with the softening process is irrecoverable, i.e., upon unloading from extension between the critical and the limiting values, the spring stiffness remains constant at the reduced value. At the limiting extension value, the area under the TS curve equals the critical fracture energy $\left(\mathcal{G}_{c}\right)$ of the interface whose value is generally derived from the test data. Works cited above addressing the interplay between the destabilizing contact interaction, and the stabilizing elastic deformations of a film and the concomitant debonding instability suggest that the threshold for undulatory separation can be modeled by a TS relation. Hui et al. [18] illustrated this for debonding between two blocks made of the same material.

In order to understand the mechanics of the peeling and demolding processes, researchers have often relied on numerical simulations. Since the use of the CZM coupled with the finite element method (FEM) by Hillerborg et al. [19] to study fracture problems, significant progress has been made in modeling interfacial debonding/delamination of a polymer interlayer [20-23]. However, there has been limited research $[8,24,25]$ on capturing progressive interfacial debonding undulations using the CZM. The formation of debonding undulations (such as fingering in a peeling problem) adds to the complexity of the mechanics of the demolding process. The development of a tool for capturing such phenomena is important for delineating the debonding process and improving our understanding of the associated mechanics. The focus of our work is to identify the role of the TS, the material and the geometric parameters in causing undulatory debonding.

The rest of the paper is organized as follows: In Section 2, we present a linear stability analysis of plane strain deformations of a semi-infinite elastomeric layer debonding from a rigid adherend pulled vertically outward. We find a non-dimensional parameter in terms of the TS and the elastic film parameters that must exceed a critical value for debonding to exhibit an undulatory morphology. In Section 3, we analyze the two-dimensional (2D) problem by the FEM to provide details of the debonding evolution and confirm the necessary condition derived by the linear stability analysis. In Section 4, we use the FEM to analyze a practical problem, namely 3D deformations when a flexible plate is peeled off a confined elastomeric layer bonded to a rigid substrate. This necessitates introducing another non-dimensional parameter in terms of the plate bending stiffness and elastomer properties. We correlate results of this problem with our learnings from Sections 2 and 3. Conclusions are summarized in Section 5.

\section{Analytical approach}

\subsection{Problem formulation}

We investigate the initiation of wavy debonding using a CZM when a rigid smooth adherend bonded to the upper surface of an elastomeric layer is pulled upwards while its lower surface stays perfectly bonded to the rigid support. Our analysis is essentially similar to those of Shenoy and Sharma [10] and Huang et al. [14] who used a linear stability approach to study instabilities in an elastic layer triggered by van der Waals forces when a rigid probe is brought near the layer. However, we use a TS relation to simulate debonding between the elastomeric layer and the upper rigid adherend. The configuration studied is schematically shown in Fig. 1. The infinitely strong perfect bonding between the lower stationary rigid adherend and the elastomer layer is modeled by constraining to zero displacements of points on the bottom surface of the interlayer. We assume that the interlayer material is isotropic, incompressible, homogeneous, and linear elastic/viscoelastic. The problem is first analyzed for a linear elastic interlayer,
Applied displacement, $\Delta$

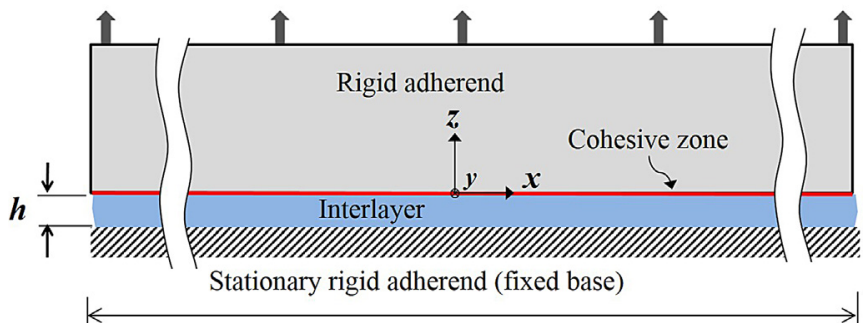

$\boldsymbol{L}$

Fig. 1. Schematic sketch of the problem studied. The width $W$ (normal to the page, not shown) is large enough to justify studying plane strain deformations in the $x z$ plane.

and subsequently for a viscoelastic layer. For the assumed form of the solution using the separation of variables with evolution in time represented by a sine (or cosine) function, the constitutive relation for a linear viscoelastic material reduces to that of a linear elastic material with the shear modulus depending upon a solution variable.

\subsubsection{Linear elastic material}

The constitutive equation for an incompressible, homogeneous and isotropic linear elastic material is $\sigma_{i j}=-p \delta_{i j}+2 \mu \varepsilon_{i j}$, where $\sigma_{i j}$ is the stress tensor (Each of the indices $i, j$ and $k$ corresponds to $x, y$, and $z$, directions), $\varepsilon_{i j}$ the strain tensor for infinitesimal deformations, $p$ the hydrostatic pressure not related to strains, and $\mu$ the shear modulus of the interlayer material. Recalling the strain-displacement relation, $\varepsilon_{i j}=\frac{1}{2}\left(\frac{\partial u_{i}}{\partial x_{j}}+\frac{\partial u_{j}}{\partial x_{i}}\right)$, the incompressibility constraint, $\varepsilon_{x x}+\varepsilon_{y y}+\varepsilon_{z z}=0$, and assuming zero body force and negligible inertial effects the equilibrium equations $\frac{\partial \sigma_{i j}}{\partial x_{j}}=0$ (repeated index $j$ is summed over $x, y$, and $z$ ) reduce to the following Navier's equations.

$$
\begin{aligned}
& \frac{\partial p}{\partial x}=\mu\left(\frac{\partial^{2} u}{\partial x^{2}}+\frac{\partial^{2} u}{\partial z^{2}}\right) \\
& \frac{\partial p}{\partial z}=\mu\left(\frac{\partial^{2} w}{\partial x^{2}}+\frac{\partial^{2} w}{\partial z^{2}}\right)
\end{aligned}
$$

Here and below we denote the displacement components $u_{x}$ and $u_{z}$ as $u$ and $w$, respectively. Perfect bond with the fixed lower rigid adherend is incorporated by setting $u$ and $w$ equal to zero at the lower interface of the interlayer. For thin confined infinitely wide incompressible films under tension, the shear stress over the central region is negligible, e.g., see Lindsey et al. [26]. Thus, boundary conditions are:

$$
\begin{aligned}
& \text { Fixed base : } u(x,-h)=w(x,-h)=0 \\
& \text { Surface tractions : }\left\{\begin{array}{l}
\sigma_{z z}(x, 0)=-p(x, 0)+2 \mu \frac{\partial w}{\partial z}(x, 0)=T(\delta) \\
\sigma_{x z}(x, 0)=\mu\left(\frac{\partial w}{\partial x}(x, 0)+\frac{\partial u}{\partial z}(x, 0)\right)=0
\end{array}\right.
\end{aligned}
$$

The interfacial normal traction $T$ is related to the displacement jump $\delta=\Delta-w(x, 0)$ by the TS relation that characterizes the interface between the interlayer and the upper adherend. Here $\Delta$ is the vertical displacement of the upper rigid adherend. We have tacitly assumed that the region of interest is far removed from the edges $x= \pm \frac{L}{2}$ and boundary conditions at these edges, not specified in Eq. (2), do not affect deformations in the interior.

2.1.1.1. Description of cohesive zone behavior at the interface. We consider a bilinear TS relation [27] for the interface, illustrated in Fig. 2, and given by Eq. (3). That is, the traction first increases linearly with the displacement jump, commonly termed the contact opening/separation, over the region OA. Point A denotes 


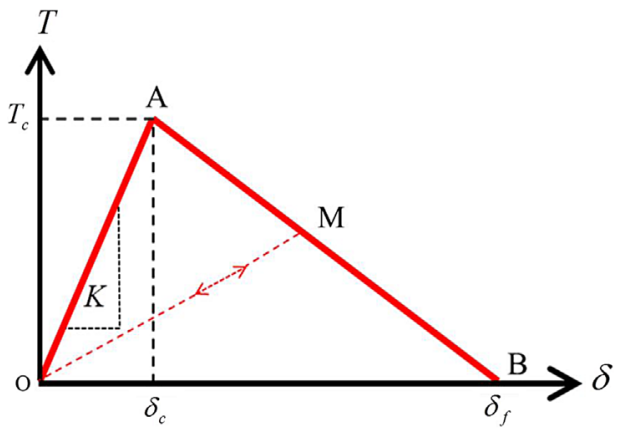

Fig. 2. A bilinear traction-separation relation.

initiation of damage/softening beyond which the traction decreases affinely with an increase in the contact opening (line $A B$ ). Should unloading occur at point $\mathrm{M}$, the traction follows the path MO. Subsequent reloading occurs along the path OMB.

$$
T(\delta)= \begin{cases}K \delta & \left(0 \leq \delta \leq \delta_{c}\right) \\ \frac{T_{c}}{\delta_{f}-\delta_{c}} & \left(\delta_{f}-\delta\right)\left(\delta_{c} \leq \delta \leq \delta_{f}\right) \\ 0 & \left(\delta_{f} \leq \delta\right)\end{cases}
$$

Point B signifies complete separation at a point on the interface. The energy release rate at debonding (i.e., the fracture energy) equals the area of the triangle OAB. That is,

$\mathcal{G}_{c}=\frac{1}{2} T_{c} \delta_{f}$

A complete description of the bilinear TS relation involves specifying the initial slope $K$, the peak traction $T_{c}$, and the fracture energy $\mathcal{G}_{c}$. In general, the value of $K$ must be large enough to not significantly alter the effective stiffness of the system and prevent interpenetration under compression. A very large value of $K$ can make the system matrices ill-conditioned when the problem is analyzed by the FEM. While $\mathcal{G}_{c}$ can be obtained directly from test data [28,29], finding values of parameters, $T_{c}$ and $\delta_{f}$, is difficult. An indirect method, frequently employed $[22,30]$, is to iteratively find values of these parameters which, when used in numerical simulations, can predict reasonably well the experimental load-displacement traces. Recently, the digital image correlation [31] and the interferometry techniques [32] have been used to measure the TS parameters.

2.1.1.2. Homogeneous solution. We note that the homogenoeous solution ( $u=0, w=0, p=$ constant) satisfies equilibrium Eq. (1) and boundary conditions (2) for $T(\delta)=-p$. The corresponding stresses are $\sigma_{z z}=\sigma_{x x}=\sigma_{y y}=-p$ and $\sigma_{z x}=0$. The pressure $p$, found from Eqs. (2a) and (3a), is given by Eq. (5). This homogeneous solution implies that pulling the upper adherend upwards will stretch the fictitious $\mathrm{CZ}$ springs while the interlayer will not deform.

$$
p=\left\{\begin{array}{lc}
-K \Delta & \left(0 \leq \Delta \leq \delta_{c}\right) \\
-\frac{T_{c}}{\delta_{f}-\delta_{c}}\left(\delta_{f}-\Delta\right) & \left(\delta_{c} \leq \Delta \leq \delta_{f}\right) \\
0 & \left(\delta_{f} \leq \Delta\right)
\end{array}\right.
$$

2.1.1.3. Non-homogeneous solution. We now explore the possibility of a non-homogeneous solution of the boundary value problem defined by Eqs. (1-3). For the deformation to identically satisfy the incompressibility constraint, we write displacements in terms of a stream function $\psi$ as

$u=\frac{\partial \psi}{\partial z}, w=-\frac{\partial \psi}{\partial x}$ where $\psi$ is a twice continuously differentiable function of $x$ and $z$. In order to find necessary conditions for the instability of the homogeneous solution $\psi=$ constant and $p=$ constant (defined by Eq. (5)), we perturb it by adding to it the non-homogeneous field: $p^{n h}(x, z)=e^{i k x} \tilde{p}(z)$ and $\psi^{n h}(x, z)=e^{i k x} \tilde{\psi}(z)$. Here the superscript $n h$ stands for non-homogeneous, $i=\sqrt{-1}$, and $k$ represents the wavenumber of the perturbation. ${ }^{1}$ The perturbed form, when substituted into equilibrium Eq. (1), yields the following system of ordinary differential equations (ODEs).

$\mu\left(\frac{d^{3} \tilde{\psi}}{d z^{3}}-k^{2} \frac{d \tilde{\psi}}{d z}\right)-k \tilde{p}=0$

$i \mu k\left(-\frac{d^{2} \tilde{\psi}}{d z^{2}}+k^{2} \tilde{\psi}\right)-\frac{d \tilde{p}}{d z}=0$

The ODEs (7) have the solution

$\tilde{p}(z)=\cosh (k z) A_{1}+i k^{2} \mu \sinh (k z) A_{2}-i \mu \sinh (k z) A_{4}$

$\tilde{\psi}(z)=\frac{\left[\begin{array}{l}i(-k z \cosh (k z)-\sinh (k z)) A_{1}+k^{2} \mu(2 \cosh (k z)-k z \sinh (k z)) A_{2}+ \\ 2 k \mu \sinh (k z) A_{3}+k \mu z \sinh (k z) A_{4}\end{array}\right]}{2 k^{2} \mu}$

where constants $A_{1}, \ldots, A_{4}$ of integration are determined from the boundary conditions. Recalling that $\Delta$ is not perturbed, $\delta=$ $\Delta-w(x, 0)=\Delta+i k e^{i k x} \tilde{\psi}(0)$. In terms of the non-homogeneous zdependent terms, boundary conditions (2) become:

Fixed base : $\tilde{\psi}(-h)=\frac{d \tilde{\psi}}{d z}(-h)=0$

Top face : $\left\{\begin{array}{l}\tilde{p}(0)+2 \mu k \frac{d \tilde{\psi}}{d z}(0)+\alpha k \tilde{\psi}(0)=0 \\ \frac{d^{2} \tilde{\psi}}{d z^{2}}(0)+k^{2} \tilde{\psi}(0)=0\end{array}\right.$

where

$\alpha= \begin{cases}-K & \left(0 \leq \delta \leq \delta_{c}\right) \\ \frac{T_{c}}{\delta_{f}-\delta_{c}} & \left(\delta_{c} \leq \delta \leq \delta_{f}\right) \\ 0 & \left(\delta_{f} \leq \delta\right)\end{cases}$

We assume that $\delta$ is increased monotonically during the debonding process. Two out of four constants in solution (8) are evaluated from boundary conditions (9a,b). Enforcing the remaining boundary conditions $(9 \mathrm{c}, \mathrm{d})$ we obtain the eigenvalue problem $[C]$ $\{A\}=\{0\}$ that has a non-trivial solution if and only if $\operatorname{det}[C]=0$. This gives the following equation for $k$ of which we seek real roots.

$f(h k)-\frac{\alpha h}{\mu}=0$

where $f(h k)=\frac{2 h k\left(1+e^{2 h k}\right)^{2}+8 e^{2 h k}(h k)^{3}}{e^{4 h k}-4 h k e^{2 h k}-1}$. Roots of Eq. (11) depend upon values of the TS parameters through their dependence upon $\alpha$, the interlayer thickness $h$, and the shear modulus, $\mu$, of the interlayer material. The function $f$ versus $h k$, plotted in Fig. 3 , has the minimum value 6.22. Thus Eq. (11) has real roots only if $\alpha h / \mu \geq 6.22$ and $\alpha$ is positive. Eq. (10) implies that the separation, $\delta$, must have values greater than $\delta_{c}$ and at most equal to $\delta_{f}$.

\footnotetext{
${ }^{1}$ The assumed perturbation, in the form of separation of variables with the complex $x$-dependent term, implies a sinusoidal variation in $x$-direction of wavenumber $k$. Any perturbation can be considered for finding necessary conditions. However, finding sufficient conditions is more challenging.
} 


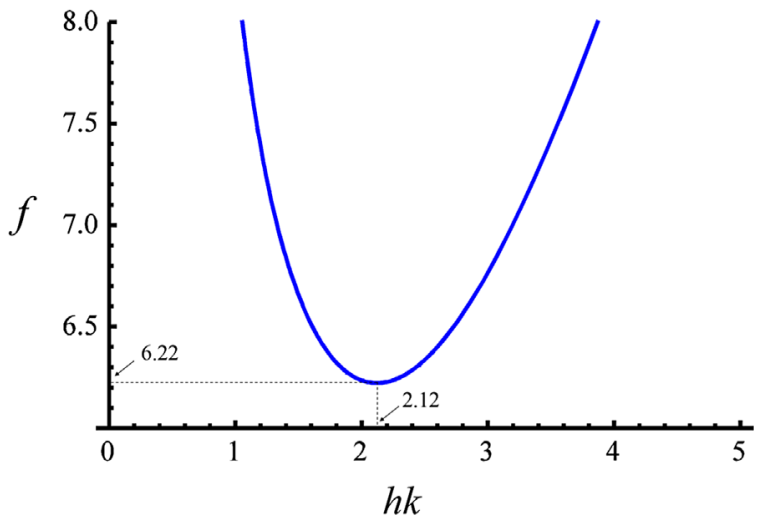

Fig. 3. The plot of function $f(h k)$. The implication is that a sinusoidal solution with wavenumber $k$ is possible when $\alpha h / \mu \geq 6.22$. Note that $f$ is a positive valued function of $h k$.

For $\alpha h / \mu>6.22$, one real root of Eq. (11) is less than 2.12 and the other greater than 2.12. The perturbed solution can be expressed as a linear combination of these two solutions. It is possible that the two perturbations will grow at different rates.

\subsubsection{Linear viscoelastic material}

For a linear viscoelastic interlayer we assume the following constitutive relation.

$\sigma_{i j}(x, z, t)=-p(x, z, t) \delta_{i j}+\int_{0}^{t} 2 \mu_{R}\left(\frac{t-s}{a_{T}}\right) \frac{\partial \varepsilon_{i j}(x, z, s)}{\partial s} d s$

Here $\varepsilon_{i j}(x, z, t)=0$ for $t \leq 0$, $\mathrm{t}$ is the present value of time and $\mu_{R}$ is the relaxation function expressed as Prony series [33], $\mu_{R}(t)=\mu_{\infty}+\sum_{i=1}^{n} \mu_{i} e^{-t / \tau_{i}}$. Here $\mu_{i}$ and $\tau_{i}$ denote, respectively, the shear modulus and the relaxation time. For discussion later, the dependence of $\tau_{i}$ upon the temperature ${ }^{2} \tilde{T}$ is included; i.e., $\tau_{i}(\tilde{T})=\tau_{i}\left(\tilde{T}_{R E F}\right) a_{T}$, where the thermal shift factor $a_{T}$ is given by the Williams-Landel-Ferry (WLF) equation: $\log _{10}\left(a_{T}\right)=\frac{-C_{1}\left(\tilde{T}-\tilde{T}_{R E F}\right)}{C_{2}+\tilde{T}-\tilde{T}_{R E F}}$, $C_{1}$ and $C_{2}$ are constants, and $\tilde{T}_{R E F}$ is the reference temperature [33]. The temperature is assumed to be uniform in the interlayer and stay constant during its deformations. Furthermore, for simplicity, we assume that the TS parameters are independent of the temperature and the loading rate.

2.1.2.1. Homogeneous solution. We note that the trivial solution ( $u=0, w=0, p=$ constant) satisfies governing equations for a viscoelastic layer provided that values of the TS parameters are independent of the rate of increase of $\Delta, \dot{\Delta}$.

2.1.2.2. Non-homogeneous solution. We add to homogeneous fields the perturbation $p^{n h}(x, z, t)=e^{i k x} \tilde{p}(z) e^{\omega t}$ and $\psi^{n h}(x, z, t)=e^{i k x} \tilde{\psi}(z) e^{\omega t}$. The real part of $\omega$ gives the growth rate of perturbations. The perturbation will grow (decay) if the real part of $\omega$ is positive (negative). Although perturbations are time-dependent, we ignore inertia forces in equations of motion because the time of interest is much larger than that taken by an elastic wave to travel through the interlayer thickness (see Appendix A). Substitution of the perturbed field into Eq. (12) gives

$\sigma_{i j}=-p \delta_{i j}+2 \bar{\mu} \varepsilon_{i j}$

where $\bar{\mu}=\mu_{\infty}+\sum_{i=1}^{N} \frac{\mu_{i} \omega \bar{\tau}_{i} a_{T}}{1+\omega \bar{\tau}_{i} a_{T}}$. Eq. (13) is the constitutive equation of the linear elastic material with shear modulus $\bar{\mu}$. For one-term Prony

${ }^{2}$ We introduce the tilde $(\sim)$ notation to distinguish temperature from traction but use the conventional notation $a_{T}$ for the thermal shift factor. series (i.e., Zener's model), $i=1$, and we set $\bar{\tau}_{i}=\bar{\tau}, \bar{\mu}=\mu_{\infty}+\frac{\mu_{1} \tilde{\omega}}{1+\tilde{\omega}}$ where $\tilde{\omega}=\omega \bar{\tau} a_{T}$. Replacing $\mu$ by $\bar{\mu}$ in Eq. (11) and solving the resulting equation for $\tilde{\omega}$, we get

$\tilde{\omega}=\frac{\phi-f}{m f+f-\phi}$

where we have set $\phi=\alpha h / \mu_{\infty}$ and $m=\mu_{1} / \mu_{\infty}$. We call $\phi$ the CZM control parameter; it equals the ratio of the interfacial softening stiffness relative to the effective long-term stiffness (modulus to thickness ratio) of the interlayer and signifies the potential importance of the former relative to that of the latter. Eq. (10) implies that $\alpha$ equals the magnitude of the slope of line $A B$ (softening stiffness) in Fig. 2.

\subsection{Results and discussion}

We recall that the function $f$ given after Eq. (11) is positivevalued (see Fig. 3), $m>0$ for a viscoelastic elastomer, and $\phi>0$ since $\alpha, h$ and $\mu_{\infty}$ are positive. Then for the growth rate $\tilde{\omega}$ to be real and positive, the following inequalities (15) must hold since the numerator and the denominator of the expression on the right hand side of Eq. (14) cannot be simultaneously negative.

$f<\phi<(m+1) f$

Eq. (14) and inequalities (15) suggest that the fastest growing wavelength corresponds to the minimum value of $f(h k)$ which is independent of values assigned to the CZM parameters and the interlayer material. Either by setting $\frac{d f}{d(h k)}=0$ or from Fig. 3, we get $h k=2.12, f(h k)=6.22$. We call values of variables for $k=2.12 / h$ critical and denote them by a subscript $c$. Thus $\lambda_{c}=(2 \pi / k)=2.96 h$, and for this value of $h k, \frac{d^{2} f}{d(h k)^{2}}>0$. Between two critical values, $\phi_{c 1}$ $\approx 6.22$ and $\phi_{c 2} \approx 6.22(1+m)$, the growth rate $\tilde{\omega}$ of perturbations is positive for all wavenumbers lying between roots of $f(h k)=\phi$, as shown in Fig. 4a in which we have plotted the growth rate as a function of the wavenumber for values of $\phi$ in the range $\left[\phi_{c 1}, \phi_{c 2}\right)$. Thus the interaction parameter $\phi$ must exceed the threshold $\phi_{c 1}$ for a spatially sinusoidal perturbation to grow. Beyond the upper critical value, $\phi_{c 2}$, of $\phi$ the growth rate becomes negative for wavenumbers lying between the two roots of $f(h k)=\frac{\phi}{m+1}$, as illustrated in Fig. $4 \mathrm{~b}$ in which we have plotted the growth rate as a function of wavenumber for a $\phi>\phi_{c 2}$. Contours in the wave number- $\phi$ plane of the normalized growth rate $\tilde{\omega}$ of perturbations for $m=3$ and $a_{T}$ $=1$ are shown in Fig. 5. For values of $\phi$ in region $I\left(\phi<\phi_{c 1}\right)$, perturbations will not grow and the adherend will snap off the interlayer when the debonding criterion is satisfied. In region II $\left(\phi_{c 1}<\phi<\phi_{c 2}\right)$, perturbations will grow with growth rate depending upon the wavenumber $k$. For a given value of $\phi$ the undulation will consist of infinitely many wavenumbers with positive growth rates, and the resulting displacement will not be a pure sine curve. In order to delineate this, we need to find amplitudes of perturbations, which is beyond the scope of the linear analysis. The numerical solution of the problem by the FEM reported in Section 3 provides details of the debond nucleation and evolution. It is possible that the wavelength with the maximum growth rate will determine the minimum spacing between adjacent undulations as stipulated by Wright and Ockendon [34] in their study of adiabatic shear bands.

It can be noted from Fig. 4a that the range of wavenumbers with positive growth rate increases as a function of $\phi$, with the wavenumber $2.12 / h$ having the fastest growth rate that is independent of $\phi$. The growth rate as a function of the wavenumber plotted in Fig. 4b illustrates an example of the behavior when $\phi>\phi_{c 2}$, i.e., region labeled III in Fig. 5. While the wavelength $\lambda_{c}$ $=2.96 \mathrm{~h}$ is no longer expected in this region, two wavelengths with 
a

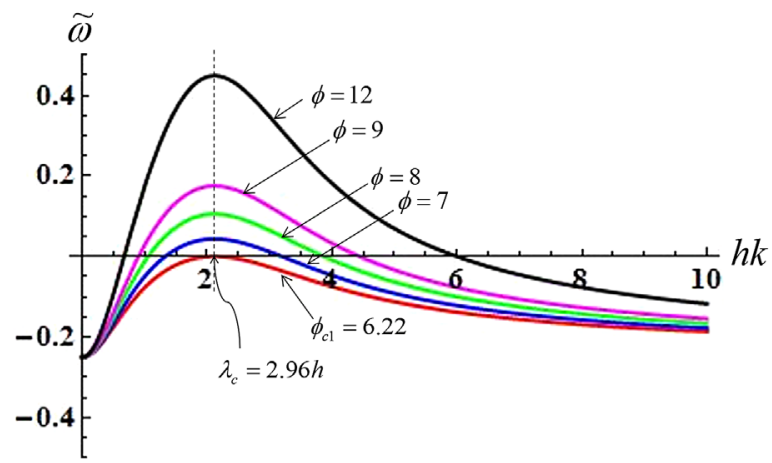

b

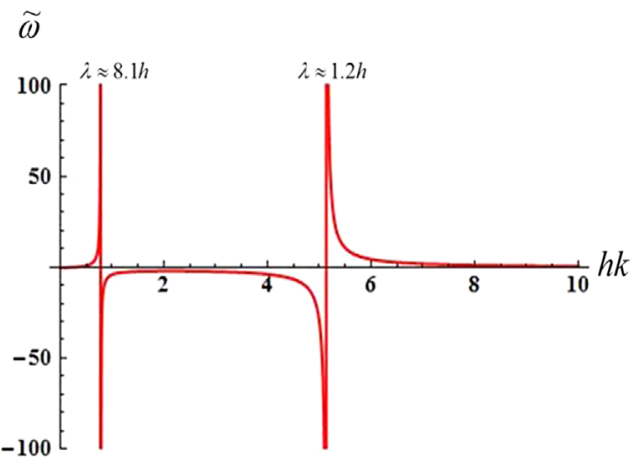

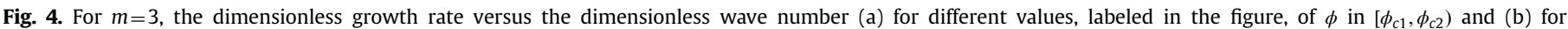

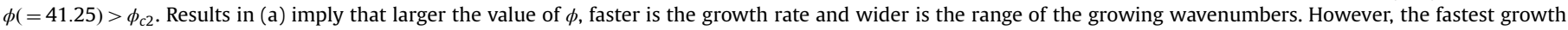

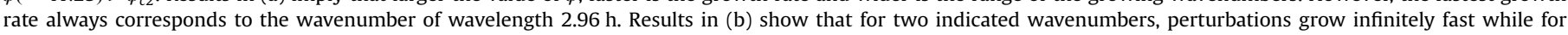
wavenumbers lying between them they decay due to their negative growth rates.

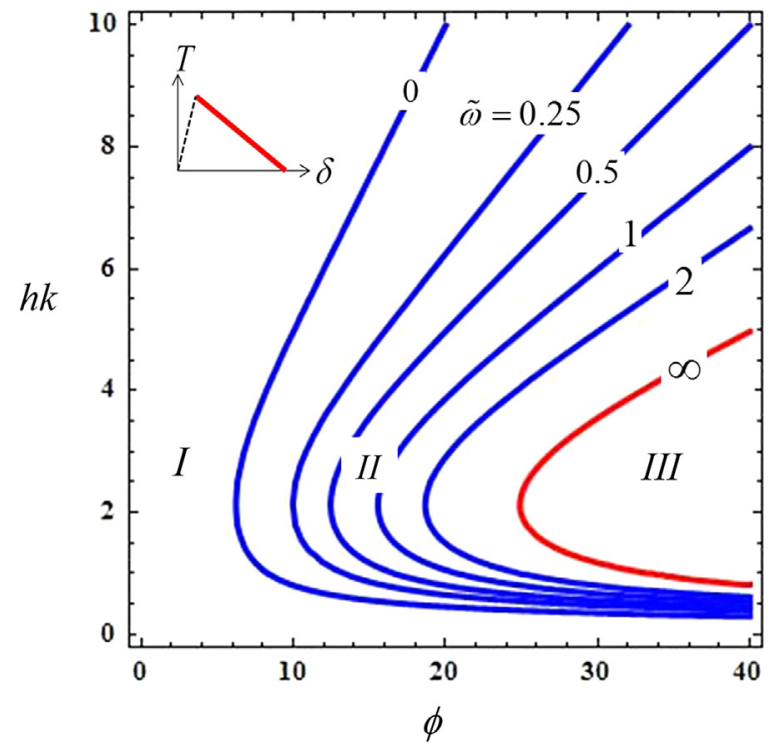

Fig. 5. Contours in the wavenumber- $\phi$ plane of the normalized growth rate $\tilde{\omega}$ of perturbations for $m=3$. Values of $\tilde{\omega}$ are indicated on the contours. These plots imply that a sinusoidal perturbation of wavenumber $h k$ will decay in regions $I$ and III and grow in region II.

infinite growth rate will be close to $\lambda_{c}=\frac{2 \pi}{\eta_{1}} h$, and $\frac{2 \pi}{\eta_{2}} h$, where $\eta_{1}$ and $\eta_{2}$ are roots of $f(\eta)=\frac{\phi}{m+1}$.

For an elastic interlayer, $\mu_{1} \rightarrow 0$ or $m \rightarrow 0$, we get $\bar{\mu} \rightarrow \mu_{\infty}$ which implies that $\phi \rightarrow f$. Thus the right hand side of Eq. (14) approaches $0 / 0$. Using L'Hôpital's rule, we obtain $\tilde{\omega} \rightarrow 0$. As depicted in Fig. 6, the region (II) with positive growth rates collapses gradually with decreasing $m$ to that for a linear elastic interlayer. In region III, it is possible that the resulting debond nucleation wavelength will depend on $\phi$.

The growth term $e^{\omega t}$ at the onset of softening in the CZM TS relation (i.e., point A in Fig. 2) can be written as $e^{\tilde{\omega} \frac{T_{C}}{K \Delta a_{T}}}$. At a given value of $\phi$, a higher value of the pulling rate $\dot{\Delta}$ implies a smaller amplitude of the sinusoidal oscillation that follows the onset of softening. Thus wavy debonding may not be experimentally discernible for very high pulling speeds. Since lowering the temperature implies increasing the effective relaxation time $\left(\bar{\tau} a_{T}\right)$, lowering temperature for fixed $\dot{\Delta}$ will have similar effect as increasing $\dot{\Delta}$. This suggests the time-temperature equivalence of wavy debonding behavior and agrees with the experimental findings of Lakrout et al. [35]. Since the normalized growth rate $\tilde{\omega}$ increases from zero to infinity as $\phi$ is varied from $f$ to $(m+1) f$ for fixed $h k$, therefore, at a given $\dot{\Delta}$ and operating temperature, $\phi$ can be increased to achieve physically discernible wavy debonding.

Recalling that $\phi=\frac{\alpha h}{\mu_{\infty}}, \quad \alpha=\frac{T_{c}}{\delta_{f}-\delta_{c}}$ (when $\delta_{c} \leq \delta \leq \delta_{f}$ ) and $K>>1$, the condition $\phi>\phi_{c 1}=6.22$ gives the following requirement for the wavy debonding to ensue.

$K_{\text {softening }}>4.15 K_{\text {elastic }}$

Here $K_{\text {softening }}=\frac{T_{c}{ }^{2}}{\mathcal{G}_{c}}$ is a measure of the slope of the softening portion of the bilinear TS relation and $K_{\text {elastic }}=\frac{E_{\infty}}{h}$, where $E_{\infty}=3 \mu_{\infty}$ is the long-term Young's modulus of the interlayer material. The quantity $K_{\text {elastic }}$ can be thought of as the long-term stiffness of the interlayer. On the other hand, $\phi>\phi_{c 2}$ leads to $K_{\text {softening }}>4.15 K^{\text {ins }}$ elastic, where $K_{\text {ins }}^{\text {elastic }}=\frac{E_{0}}{h}$ and $E_{0}=3\left(\mu_{\infty}+\mu_{1}\right)$ is the instantaneous Young's modulus of the interlayer material. Eq. (16) suggests that for wavy debonding to ensue, the stiffness (units: $\mathrm{N} / \mathrm{mm}^{3}$ ) of the interface during the softening regime must be 4.15 times the long-term stiffness of the interlayer. Thus the TS parameters must satisfy this constraint for wavy debonding. If the softening stiffness (divided by 4.15) lies between the long-term and the instantaneous stiffness of the elastomeric layer, the dominant wavelength is expected to be independent of the CZM parameters.

Eq. (16) provides an interpretation of the debonding instability in terms of the CZM parameters. It stems from the interfacial traction decreasing with an increase in the interfacial separation, similar to what previous researchers $[10,14]$ stated in terms of the distance-dependent van der Waals forces acting at the interface that trigger instability when a rigid adherend approaches an elastic film. Our results imply that the elastomeric layer thickness and the interfacial adhesion can be selected to either avoid or produce wavy debonding. Similarly, given the TS relation and values of parameters of the interlayer material, one can discern if the necessary condition for undulations to occur is satisfied.

One needs to analyze the nonlinear problem to study the evolution of debonding. In the following section, we use the FEM to analyze plane strain deformations of the interlayer material with the bilinear TS relation and furthermore ascertain (i) the validity of Eq. (16), and (ii) the effect of $\phi$ on the spacing between the adjacent debonding undulations. Advantages of the FE work over the analytical work include no specific form of perturbations, using the finite size specimen, considering boundary conditions at specimen edges, not assuming a-priori the deformation field, accounting for mixed-mode debonding evolution, and the capability to simulate material and geometric nonlinearities. 
a

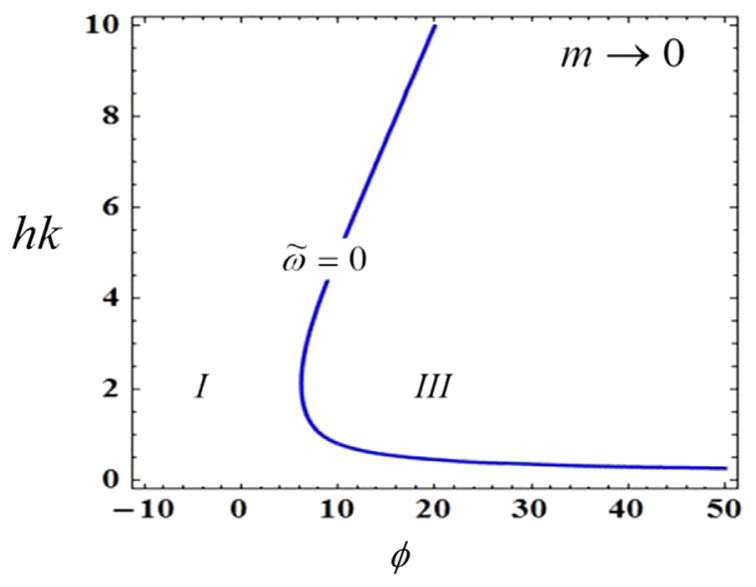

b

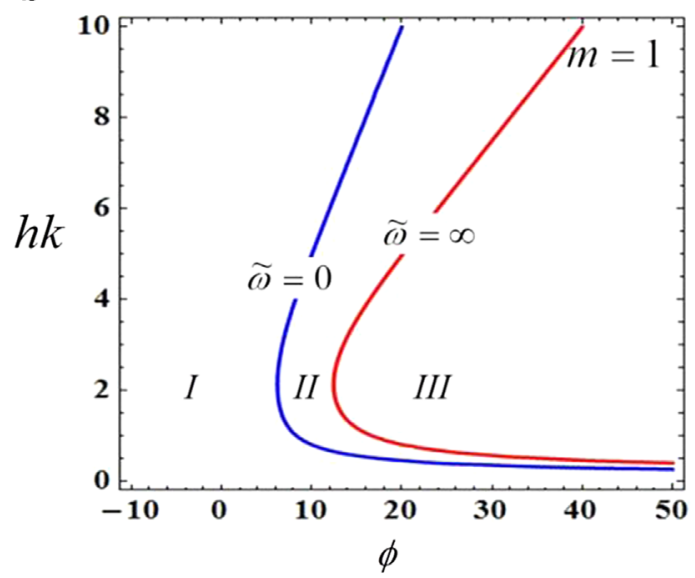

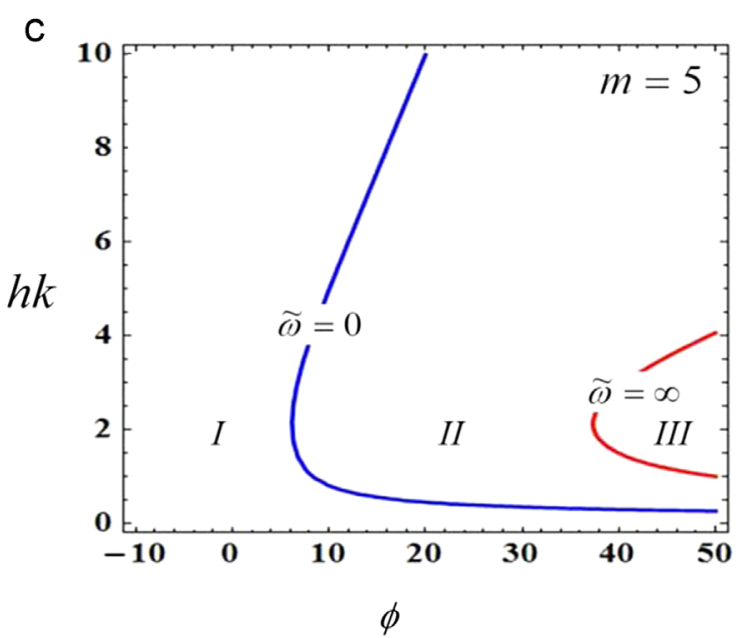

Fig. 6. Effect of $m$ : (a) elastic limit with two possible wavenumbers, (b) three regions for $m=1$, and (c) three regions with a wider region $I$ for $m=5$.

\section{Debonding of elastomeric layer from a rigid adherend by the FEM}

\subsection{Approach}

The problem studied in Section 2 is now analyzed by the FEM using the commercial software ABAQUS/Standard [36] . To be consistent with the assumption made in Section 2 that the system is infinitely wide in the $x$ direction, we use a large $L / h$ ratio $^{3}$. The FE mesh and the prescribed boundary conditions are shown in Fig. 7. Five 4-node plane strain elements with hybrid formulation (CPE4H) are placed through the thickness of the interlayer. In order to characterize well the spacing between adjacent undulations, 20 elements are placed over the expected characteristic length of the undulation spacing, i.e., $3 h$. Results for three different FE meshes are included in Appendix C. A uniform vertical displacement, $w=\Delta$, is applied at the rate of $1 \mathrm{~mm} \mathrm{~s}^{-1}$ on the top surface of the rigid adherend while its lower smooth (frictionless) surface is bonded to the top surface of the interlayer via the CZM interaction and the bilinear TS relation [36] with mode-independent values of TS parameters ${ }^{4}$. The bottom surface of the interlayer is held stationary. The remaining bounding surfaces of the interlayer are assumed to be traction free. To alleviate numerical

\footnotetext{
${ }^{3}$ Dimensions of the elastomeric layer used in most of our FE simulations are $L=23 \mathrm{~mm}, h=0.25 \mathrm{~mm}$. The size of the rigid adherend is taken to be $25 \mathrm{~mm} \times 10 \mathrm{~mm}$.

${ }^{4}$ We assume mode-independent values of the initial slope, the peak traction, and the fracture energy; see [36] for details of a mixed mode bilinear TS relation.
}

instabilities, the "Damage Stabilization" option in ABAQUS is used. The value of the stabilization parameter was gradually reduced to $10^{-8}$ and further decrease in this value was found not to affect the computed results. The interlayer material is modeled either as linear elastic or linear viscoelastic with one term in the Prony series. For the linear viscoelastic material, unless otherwise mentioned, values assigned to the material parameters are: $\tau a_{T}=10^{-5} s$ and $m=3$. Numerical experiments are conducted by varying the parameter $\frac{K_{\text {softening }}}{K_{\text {elastic }}}$ using several combinations of values of the interlayer modulus, $\mu_{\infty}$ or $\mu$, thickness, $h$, and cohesive zone parameters, $K, T_{c}$ and $\mathcal{G}_{c}{ }^{5}$.

\subsection{Results and discussion}

Wavy debonding is predicted, as shown in Fig. 8, at sufficiently large values of $K_{\text {softening. }}$. The critical $K_{\text {softening }}$ values plotted in Fig. 9 versus the elastic stiffness of the interlayer agree with those given by Eq. (16). The predicted debonding nucleates periodically ${ }^{6}$ along the $x$ direction implying long debonding channels along the $y$-direction

\footnotetext{
${ }^{5}$ As an example, values of the parameters for the simulation with $K_{\text {softening }}$ $\mid K_{\text {elastic }}=4.15$ are: $h=0.25 \mathrm{~mm} \quad \mu_{\infty}=0.2 \mathrm{Mpa}, \quad m=3, t a_{T}=10 \mu \mathrm{s}, K=10^{6} \mathrm{~N} /$ $\mathrm{mm}^{3}, T_{c}=0.04 \mathrm{MPa}$, and $\mathcal{G}_{c}=0.166 \mathrm{~N} / \mathrm{m}$.

${ }^{6}$ The inhomogeneity in the distribution of interfacial tractions, singular at the corners [37-40] triggered by the free-edges of the elastomer layer and the adjoining traction-free portion of the adherend likely acts as the perturbation to the homogeneous solution over the central region. If horizontal displacements at the free edges of the interlayer are constrained, then the undulatory debonding is not predicted. We have not attempted to capture the correct order of singularity at the corners. However, our key results are insensitive to further refinement of the FE mesh.
} 


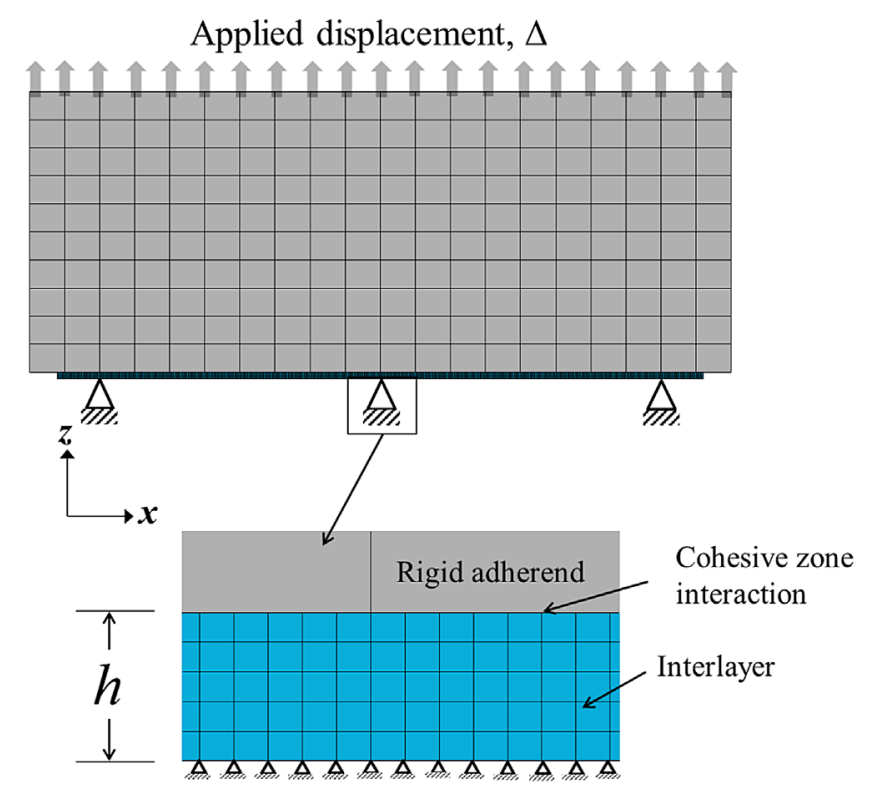

Fig. 7. Details of the FE mesh and boundary conditions. Applied zero tractions in directions of the unconstrained displacement components are not shown.

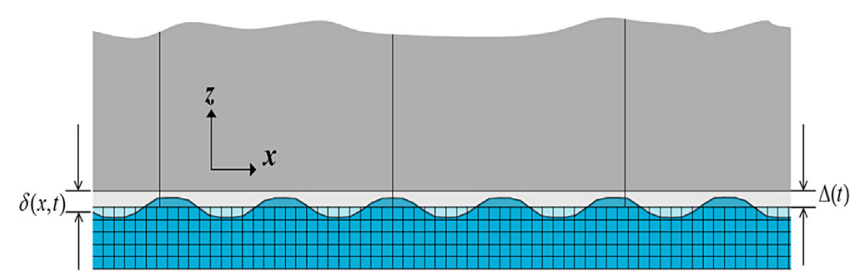

Fig. 8. Deformed and undeformed configurations when a wavy debonding is predicted by the model. The FE mesh is shown only on the undeformed configuration. The gap between the adherend and the interlayer is due to the displacement jump at the interface. Debond occurs only when this jump exceeds its prescribed limiting value.

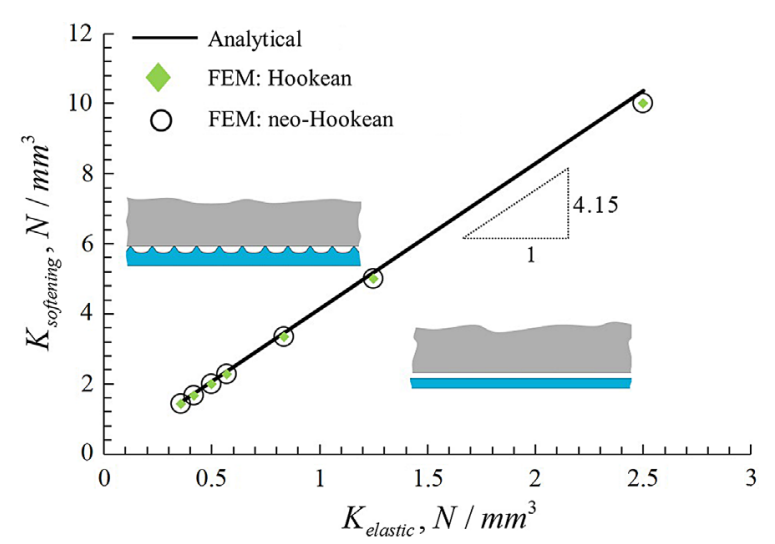

Fig. 9. The map illustrating that the quantity $T_{c}{ }^{2} / \mathcal{G}_{c}$ must exceed approximately $4.15\left(E_{\infty} / h\right)$ for a wavy debond to ensue. This plot was generated for $m=3, \tau=$ $10^{-5} \mathrm{~s}$, and $\dot{\Delta}=1 \mathrm{~mm} / \mathrm{s}$.

because of the plane strain assumption. However, in probe tack tests [7] and contact experiments [2], debonding is found to nucleate periodically along both the $x$ - and the $y$-directions. Motivated by the work of Huang et al. [14], we speculate that the condition for the onset of wavy debonding remains unchanged for 3D deformations of the interlayer. The simulated debonding evolution, in general, consists of the nucleation of interfacial cavities at a characteristic spacing, expansion of cavities and lateral propagation of each cavity until the adherend separates from the elastomeric layer as cavities coalesce.
Such debonding behavior in probe tack tests has been reported by Lakrout et al. [7]. Two examples of the evolution of wavy contact opening are shown in Fig. 10 for $\phi=8.25$ and $\phi=41.25$. The discrete Fourier transform method (available in the software MATHEMATICA [41]) is used to extract the dominant wavelength of the debond nucleation. Results are plotted in Fig. 11 for a range of $\phi$ values for an elastic interlayer $(m \rightarrow 0)$, and two viscoelastic interlayers with $m=3$ and 25 . The dominant wavelength is close to $3 \mathrm{~h}$ and independent of $\phi$ when $\phi$ lies in region II. This agrees with the value derived from the above analytical work and experimental findings of Mönch and Herminghaus [2]. For both the elastic and the viscoelastic layers, the wavelength to thickness ratio for $\phi$ near region III is found to be larger and dependent on $\phi$ than that when $\phi$ is near region II. It is noteworthy that at the larger $\phi$ value, shapes of the nucleated cavities change before they coalesce as was computed by Sarkar et al. [24]. Our numerical experiments suggest that the response becomes more mesh-dependent as $\phi$ is increased (see Appendix C).

Previous studies [2,9] suggesting that the characteristic wavelength is independent of the adhesion seem to contradict at first sight our results for large values of $\phi$. For typical experimental $[5,29]$ values of $m=10^{3}, \mathcal{G}_{c}=0.04-0.2 \mathrm{~J} / \mathrm{m}^{2}, \mu_{\infty}=0.2-2 \mathrm{MPa}$, and $h=40-400 \mu \mathrm{m}$, we get $T_{c}>2.5 \mu_{\infty}$ in the proximity of $\phi=\phi_{c 2}$. Such large values of the critical traction imply probable onset of cohesive debonding mechanisms such as bulk cavitation [42] that occurs when $(-p) \geq 2.5 \mu_{\infty}$. This suggests that the $\phi$ values for test conditions [2,9] that exhibited pure interfacial separation were well below $\phi_{c 2}$ and, therefore, the dominant wavelength was indeed independent of the adhesion.

Results depicted in Fig. 9 for the interlayer material modeled as neo-Hookean do not exhibit any significant difference in the threshold softening stiffness because strains induced in the elastomer layer when softening ensues are negligible for the large value of $K$ used.

Results summarized in Table 1 indicate that wavy debonding was not predicted when either $\dot{\Delta}=100 \mathrm{~mm} / \mathrm{s}$ or a higher value of the relaxation time $\tau a_{T}$ was used for a given value of $\phi$. However, a lower value of $\tau a_{T}$ or a higher value of $\phi$ at $\dot{\Delta}=100 \mathrm{~mm} \mathrm{~s}^{-1}$ resulted in wavy debonding. These results qualitatively confirm the time-temperature equivalence discussed at the end of Section 2.

\section{Analysis of 3D deformations for peeling of a flexible plate off a soft elastomeric layer by the FEM}

\subsection{Approach}

Results presented in the preceding two sections imply that values of parameters in the TS relation for the interface between an elastomeric layer and the rigid substrate, the applied pulling rate and the characteristic relaxation time determine whether or not a wavy debond will occur. In order to delineate fingering instability [4,5,9] for a deformable upper adherend, we study progressive crack propagation in the configuration of Fig. 12 that resembles the test set up often used to characterize interfacial adhesion and/or study mechanics of interfacial separation $[5,29,43]$. Major differences between this problem and those studied above include bending and stretching deformations of the upper adherend.

We model the plate/adherend and the interlayer of dimensions, respectively, $20 \times 30 \times 1 \mathrm{~mm}^{3}$ and $20 \times 25 \times \mathrm{h} \mathrm{mm}^{3}$. Deformations of the interlayer may be large but those of the adherend plate are assumed to be infinitesimal. Furthermore, inertial effects are neglected. We use values of the material parameters given by Murray [43] who experimentally observed fingerlike debonding during the peeling of a glassy polymer plate from a hydrogel interlayer in a wedge test. The plate material is modeled as homogeneous and isotropic Hookean 

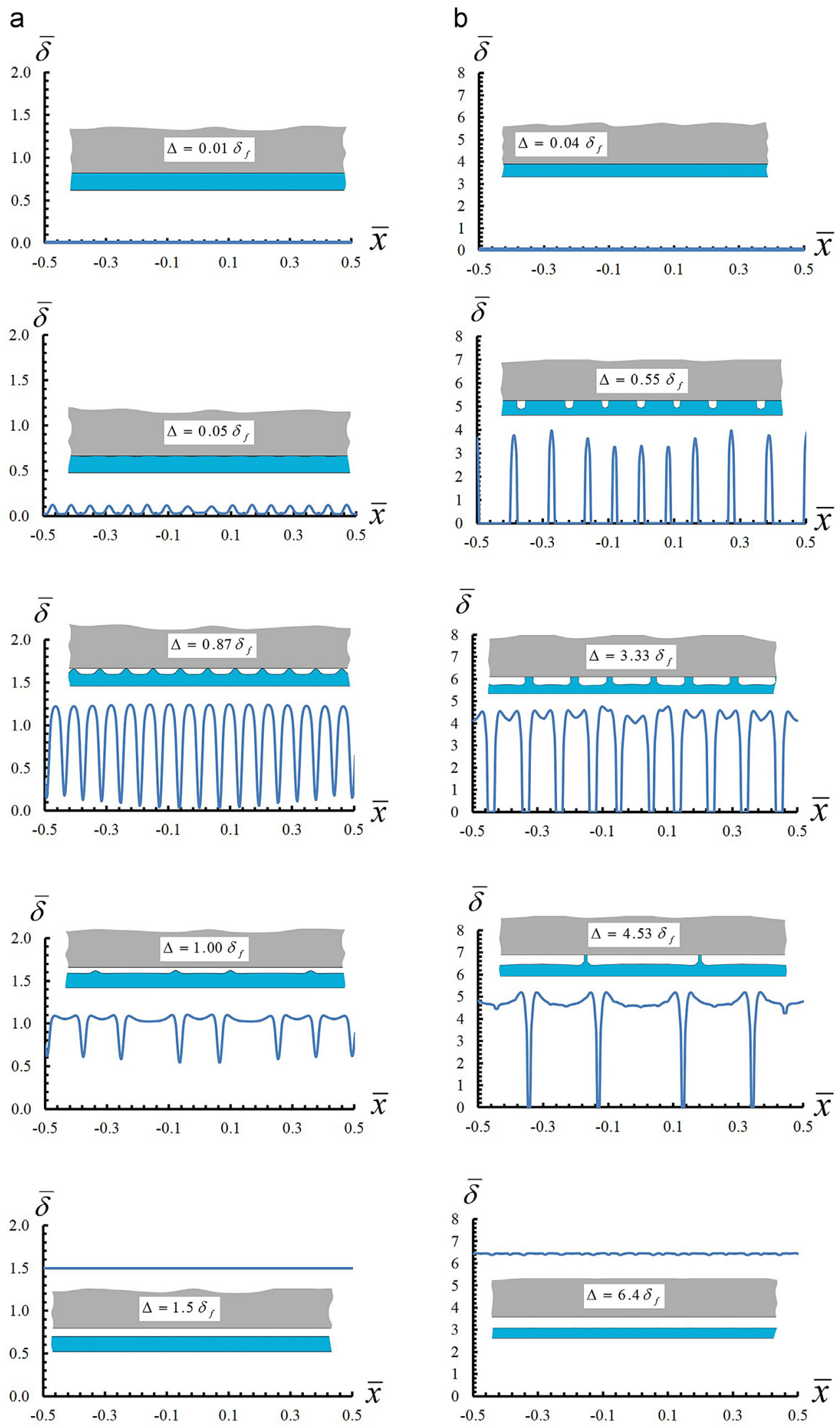

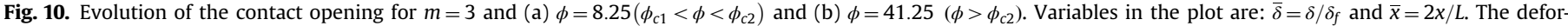

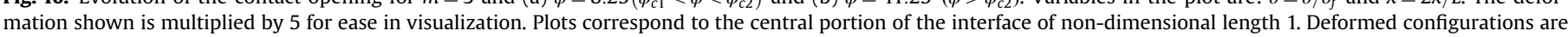
for the interface portion of non-dimensional length 0.66 . 
with Young's modulus, $E_{\text {plate }}=2.1 \mathrm{GPa}$ and Poisson's ratio $=0.4$. However, we also compute results for different values of $E_{\text {plate }}$. The material for hydrogel interlayer is modeled as isotropic, homogeneous and incompressible with the constitutive relation (17) (e.g., see Simo [44]) implemented in ABAQUS [36].

$\boldsymbol{\sigma}(t)=-p \mathbf{I}+a_{1} \boldsymbol{B}+a_{2} \boldsymbol{B}^{2}+\operatorname{dev}\left[\int_{0}^{t} \dot{g}_{R}\left(\frac{s}{a_{T}}\right) \mathbf{F}_{t}^{-1}(t-s) \boldsymbol{\sigma}_{0}^{D}(t-s) \mathbf{F}_{t}^{-T}(t-s) d s\right]$

Here $a_{1}$ and $a_{2}$ are material constants, $\boldsymbol{B}$ the left Cauchy-Green tensor, $g_{R}$ the normalized relaxation modulus expressed as Prony $\operatorname{series}\left(g_{R}(t)=\frac{\mu_{R}(t)}{\mu_{R}(0)}\right), \operatorname{dev}(A)=A-\frac{1}{3} \operatorname{tr}(A) I, \operatorname{tr}(A)=$ sum of the diagonal elements of the matrix $A$ when its components are written with respect to an orthonormal basis, $I=$ the identity matrix, $\sigma_{0}{ }^{D}$ the instantaneous deviatoric Cauchy stress, and $\mathbf{F}_{t}(t-s)$ the deformation gradient at time $(t-s)$ with respect to the configuration at

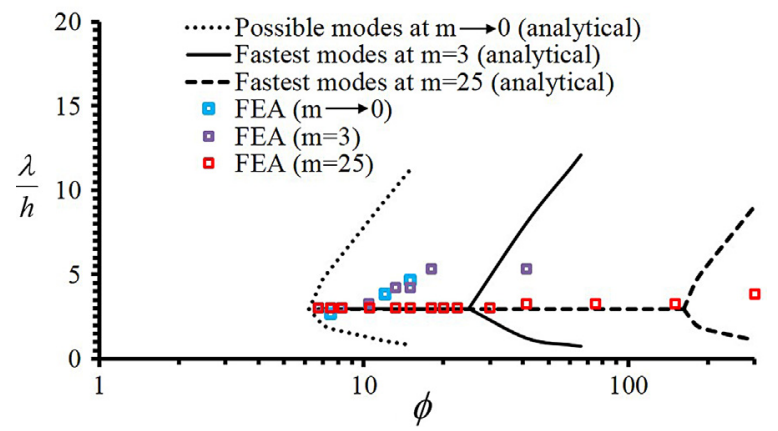

Fig. 11. Effect of $\phi$ on the dominant wavelength (normalized by thickness) of the debonding undulation. time $(t)$, defined as $\mathbf{F}_{t}(t-s)=\frac{\partial \mathbf{x}(t-s)}{\partial \mathbf{x}(t)}$ with $\mathbf{x}$ giving the current position of a material point. Based on experimental results [45], the instantaneous elastic response is assumed to be neo-Hookean,

Table 2

Effect of the TS parameters (for plate rigidity $=2.1 \mathrm{Nm}$, interlayer thickness $=250$ $\mu \mathrm{m}$, and pulling rate $=1 \mathrm{~mm} / \mathrm{s}$ ), which evince that wavy undulations vanish when the interfacial softening is increased relative to the interlayer stiffness (lower effective stiffness at a higher temperature due to viscoelasticity).

\begin{tabular}{rlll}
\hline$\tilde{T}\left({ }^{\circ} \mathrm{C}\right)$ & $T_{c}(\mathrm{MPa})$ & $\mathcal{G}_{c}\left(\mathrm{~J} / \mathrm{m}^{2}\right)$ & Type of debonding front \\
\hline 55 & 0.04 & 0.2 & Fingerlike undulations \\
55 & 0.004 & 0.2 & No fingerlike undulation \\
-30 & 0.04 & 0.2 & No fingerlike undulation \\
\hline
\end{tabular}

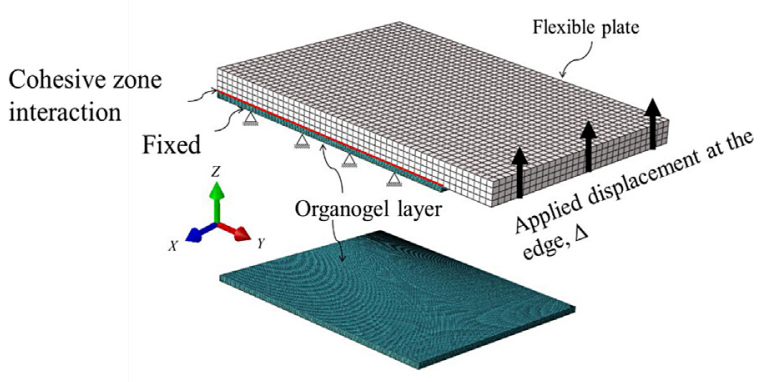

Fig. 12. The FE mesh and boundary conditions with zero tractions along unconstrained displacement components not exhibited.

Table 1

Qualitative evidence of the time-temperature equivalence in predictions from the model.

\begin{tabular}{|c|c|c|c|}
\hline \multicolumn{4}{|c|}{$\Delta=14 \mu \mathrm{m}$} \\
\hline$\phi$ & $\dot{\Delta}(\mathrm{mm} / \mathrm{s})$ & $\tau a_{T}(\mu s)$ & Debonding (Deformations exaggerated by a factor of 10 ) \\
\hline 7 & 1 & 10 & \\
\hline 7 & 100 & 10 & \\
\hline 7 & 1 & 1000 & \\
\hline 7 & 100 & 0.1 & 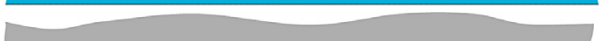 \\
\hline 28 & 100 & 10 & 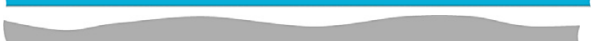 \\
\hline
\end{tabular}


a

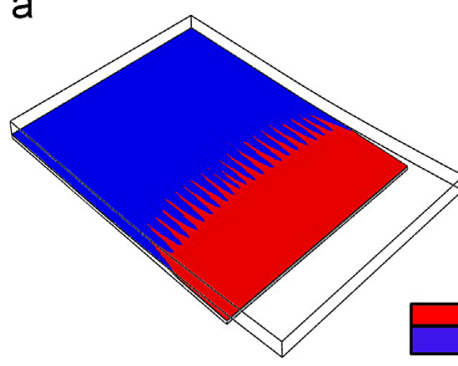

b

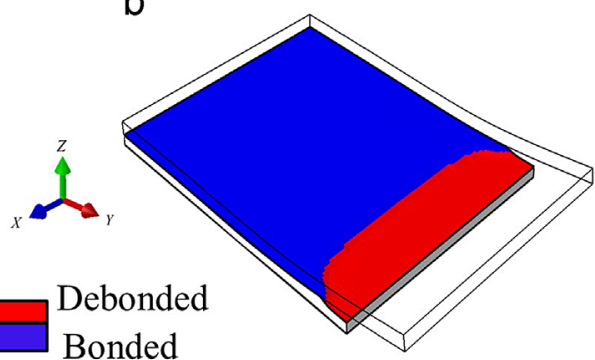

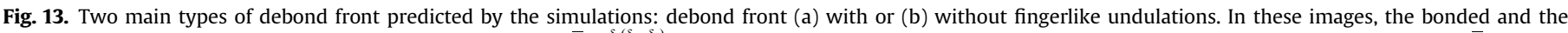

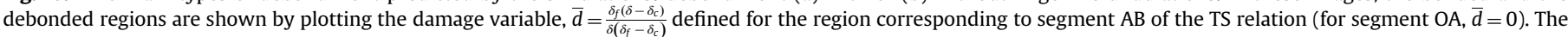

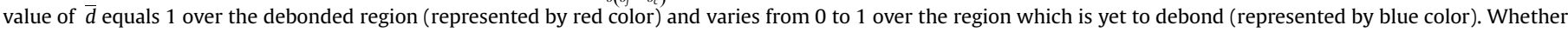

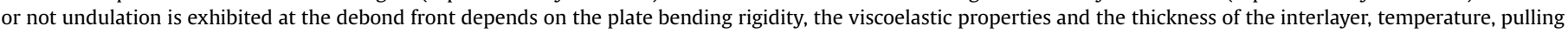
rate and values of the TS parameters. (For interpretation of the references to color in this figure legend, the reader is referred to the web version of this article.).

i.e., $a_{2}=0$, and $a_{1}=$ twice the constant shear modulus of the interlayer material. The Prony series parameters determined experimentally [45] at the reference temperature of $45^{\circ} \mathrm{C}$ are listed in Table B1. The WLF constants at the reference temperature $\tilde{T}_{R E F}=45^{\circ} \mathrm{C}$ are $\mathrm{C}_{1}=4.57^{\circ} \mathrm{C}$ and $\mathrm{C}_{2}=142.2^{\circ} \mathrm{C}$.

We assume a mixed-mode bilinear TS relation [36] with modeindependent values of the initial slope $\left(K=10^{6} \mathrm{MPa} / \mathrm{mm}\right)$, the peak traction $\left(T_{c}=0.04 \mathrm{MPa}\right)$, and the fracture energy $\left(\mathcal{G}_{c}=\right.$ $\left.0.2 \mathrm{~J} / \mathrm{m}^{2}\right)$. The value of the damage stabilization parameter for alleviating numerical instabilities was gradually reduced to $10^{-10}$ until a further decrease in this value did not affect computed results.

The boundary conditions and the FE mesh are shown in Fig. 12. The three displacement components of points on the lower face of the interlayer are set equal to zero to simulate its infinitely strong bonding to the stationary rigid adherend. The same vertical displacement $\Delta$ is applied at $1 \mathrm{~mm} \mathrm{~s}^{-1}$ to all nodes on the plate right edge. Tractions on the other bounding surfaces and tangential tractions on the right edge are null.

The eight-node brick elements (C3D8R) with reduced integration and the default hourglass control option have been used for the cover plate. Hybrid elements (C3D8RH) ${ }^{7}$ have been employed for the incompressible interlayer. The interlayer region is discretized into 5 (thickness) $\times 150$ (width) $\times 100$ (length) uniform FE mesh. This FE mesh is reasonably fine as the characteristic spacing between adjacent undulations is expected [5] to be $\lambda \approx 3 h-4 h=$ $\sim 1 \mathrm{~mm}$ for the smallest interlayer thickness used in the study. In order to demonstrate the effect of the CZM parameters on the debonding characteristics, we have not obtained a fully converged solution by successively refining the $\mathrm{FE}$ mesh since qualitative features of progressive debonding remained independent of the FE mesh.

\subsection{Results and discussion}

In general, the debond front is either fingerlike or straight as illustrated in Fig. 13. An example of the evolution of the fingerlike debonding process is shown in Fig. 14 in which we have plotted contours of the contact opening $\delta$ at three different times for a plate of bending rigidity, $D=\frac{E_{\text {plate }} h_{\text {plate }}{ }^{3}}{12\left(1-v^{2}\right)}=21 \mathrm{Nm}(v=$ Poisson's ratio, $h_{\text {plate }}=$ the plate thickness) and an interlayer of thickness $250 \mu \mathrm{m}$.

\footnotetext{
${ }^{7}$ For the 3D simulations, the overall energy balance applied to the ABAQUS output gave a discrepancy of about 20\%. Additional numerical experiments revealed that this was due to our using the default value of the hourglass control. The use of selective reduced integration or other hourglass control options satisfied the energy balance within $1 \%$ error but triggered unphysical oscillations at the contact surface. The physically meaningful results computed with the default hour glass control and their agreement with the literature results obtained by other methods provide credence to results reported here.
}

Computed values of $\delta>\delta_{f}$ are represented in red color at right ends of illustration. As experimentally observed [5,9], the development of fingers is preceded by the nucleation of debonds spaced at approximately the same distance as that between the fingers that will ensue. Decaying plate displacement, undulatory debonding, and lateral propagation of debonds give rise to what resembles fingers at the debond front.

Progressive debonding was also simulated for a plate with $D=$ $2.1 \mathrm{Nm}$ and interlayers thicknesses $250 \mu \mathrm{m}, 500 \mu \mathrm{m}, 600 \mu \mathrm{m}$ and $750 \mu \mathrm{m}$. The spacing between the adjacent fingers, computed using the discrete Fourier transform method (using MATHEMATICA [41]), versus the interlayer thickness is plotted in Fig. 15a. We note that the computed spacing increases with an increase in the interlayer thickness and reasonably agrees with experimental findings of Ghatak and Chaudhury [9] who reported the approximate relation: $\lambda \approx 4 h$. We note that for the highest thickness $(750 \mu \mathrm{m})$ simulated, fingerlike debonding was not predicted. However, debonding exhibited a fingerlike front for a plate of higher flexural rigidity $(7.5 \mathrm{Nm})$, consistent with the requirement of a threshold lateral confinement $\left(D / \mu h^{3}\right)^{1 / 3}$, expressed as the ratio of the two length scales, $(D / \mu)^{1 / 3}$ and $h[9,12]$. Simulations conducted for different values of $D$ and a constant interlayer thickness $(250 \mu \mathrm{m})$ revealed that the length of the fingers increased monotonically with the quantity $(D / \mu)^{1 / 38}$ which represents the characteristic stress decay distance for a flexible plate bonded to an elastomeric foundation [46]. The linear fit to the computed values depicted in Fig. 15b is close to that obtained by Ghatak and Chaudhury [9] for a different material system. We believe that this quantitative agreement in the finger amplitudes is coincidental because the softening zone length (in the $y$-direction) should also depend on the interfacial adhesion. We found that the dominant frequency of undulation is relatively insensitive to the plate rigidity, consistent with findings of [9].

In order to qualitatively demonstrate the concept that the CZM parameters for confined interlayers determine whether or not contact separation resulting from tension is wavy, additional simulations were conducted by varying the TS parameters and the operating temperature by keeping the thickness of the interlayer, the plate rigidity and the pulling rate constant. The key parameters used in these simulations and the results are summarized in Table 2. For $T_{c}=0.04 \mathrm{MPa}$, setting the temperature equal to $-30^{\circ} \mathrm{C}$ results in larger relaxation times following the WLF equation, and a fingerlike debond front was not predicted. Similarly, a fingerlike

\footnotetext{
${ }^{8}$ The shear modulus of the interlayer at time $t$ and temperature $\tilde{T}$ was estimated as $\mu(t, \tilde{T}) \approx \mu_{R}\left(\frac{t}{a_{T}}\right)=\mu_{\infty}+\sum_{i=1}^{N} \mu_{i} e^{-\frac{t}{a_{T} \tau_{i}}}$
} 


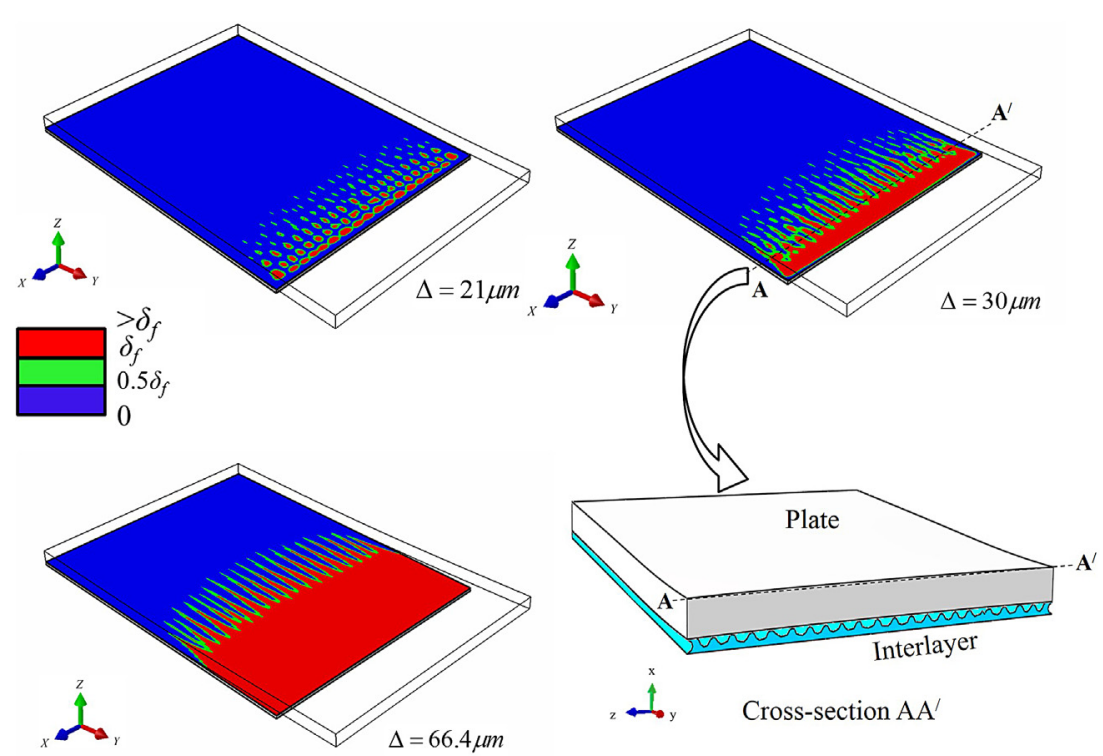

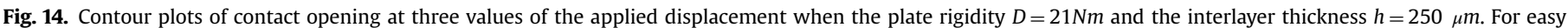
visualization, deformations are exaggerated by a factor of 10 and by a factor of 12 for the cross-section $\mathrm{AA}^{\prime}$ illustrating wavy debonding.
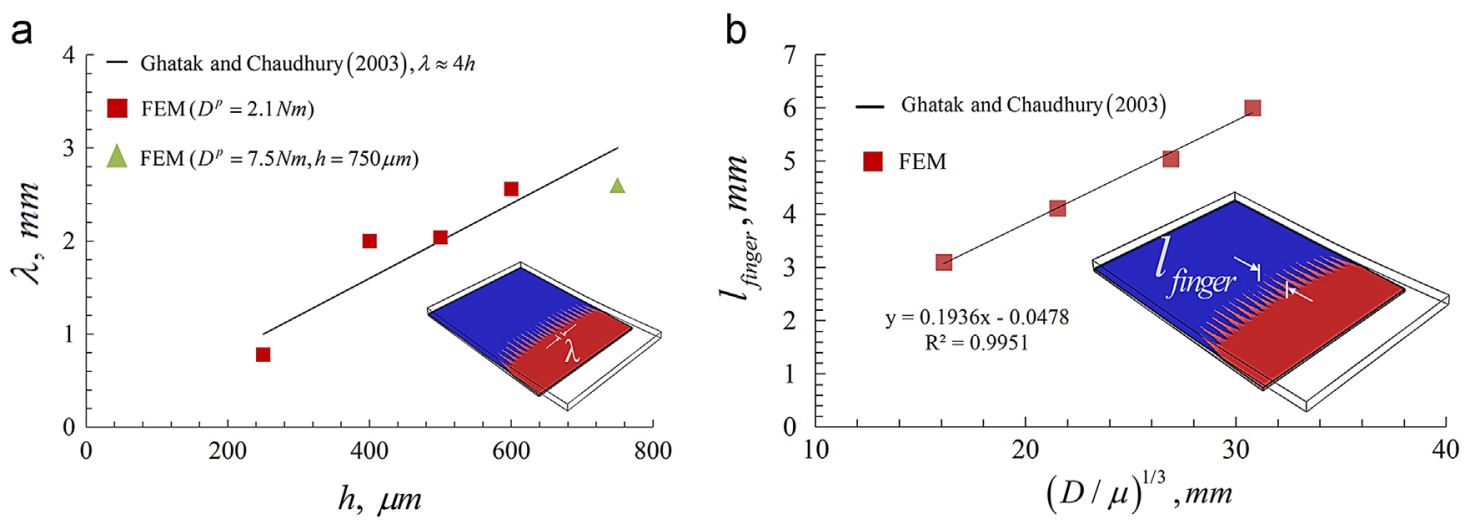

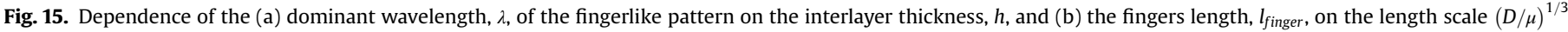

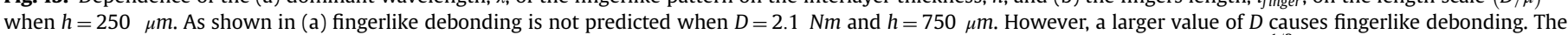

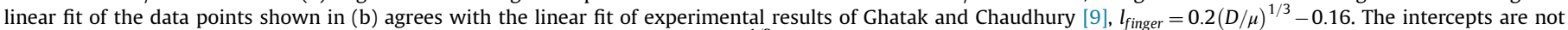
important since undulatory debond fronts do not occur for small values of $(D / \mu)^{1 / 3}$.

front was not predicted when $T_{c}=0.004 \mathrm{MPa}$ was used for the operating temperature of $55^{\circ} \mathrm{C}$.

Our computed results suggest that the debonding behavior during peeling a flexible plate off an elastomeric layer constrained to a rigid base is dictated by both the lateral confinement $\left(D / \mu h^{3}\right)^{1 / 3}$ and the adhesion parameter, $T_{c}{ }^{2} h / E_{\infty} \mathcal{G}_{c}$. A detailed analysis probing their collective role in the debonding evolution will be undertaken in a future work.

\section{Conclusions}

We have studied debonding of a confined elastomer layer from an adherend using the cohesive zone model (CZM) and the bilinear traction-separation (TS) relation for the interaction between the elastomer layer and the adjoining adherend. The stability analysis of the homogeneous solution (null displacements and constant hydrostatic pressure) of plane strain deformations of the elastomer and the analysis of deformations by the finite element method (FEM) have enabled us to conclude that a necessary condition for a wavy/undulatory debonding to ensue is $\frac{T_{c}{ }^{2} h}{\mathcal{G}_{c} E_{\infty}}>4.15$, where $T_{c}$ is the peak traction and $\mathcal{G}_{c}$ the fracture energy in the TS relation, $h$ the thickness and $E_{\infty}$ the long-term Young's modulus of the elastomeric layer modeled as linear viscoelastic. This result can help design a material system for avoiding wavy debonding by choosing thickness of the soft adhesive and/or altering the interfacial softening by suitable surface treatment. It also serves to tailor the TS relation parameters for simulating spatially undulatory debonding evolution. The linear stability analysis also predicts that if undulations occur, their dominant wavelength is close to $3 h$, when $T_{c}{ }^{2} / 4.15 \mathcal{G}_{c}$ lies between $E_{\infty} / h$ and $E_{0} / h$ where $E_{0}$ is the instantaneous modulus of the elastomeric layer. Analysis of the problem using the finite element method (FEM) provides details of the interfacial debonding evolution and sheds light on the effects of pulling rate and temperature.

We have also analyzed using the FEM three-dimensional deformations of a thin elastomeric interlayer (perfectly bonded to a rigid base) when a flexible plate is peeled from it by applying vertical displacements to points on one edge. This analysis predicts the progressive debonding with a fingerlike front is controlled by both the lateral confinement $\left(D / \mu h^{3}\right)^{1 / 3}$ and the adhesion 
parameter, $\phi$, where $D$ equals the plate bending rigidity and $\mu$ the interlayer shear modulus at the operating temperature.

\section{Acknowledgments}

The authors thank the department of Biomedical Engineering and Mechanics and Institute of Critical Technology and Applied Sciences (ICTAS), Virginia Tech for the use of its facilities and Macromolecules and Interfaces Institute (MII), Virginia Tech for fostering interdisciplinary research in the adhesion area. The valuable feedback provided by the reviewers on an earlier version of the manuscript is deeply appreciated.

\section{Appendix A}

Using the non-dimensionalization $X=x / h, Z=z / h, U=u / h, W$ $=w / h, P=p / \bar{\mu}$ and $T=t / t_{\text {int }}$ where $t_{\text {int }}=\delta_{f} / \dot{\Delta}$ is the time of interest, equations of motion for the interlayer become

$\frac{\partial^{2} U}{\partial T^{2}}+\left(\frac{t_{\text {int }}}{t_{\text {wave }}}\right)^{2}\left(-\frac{\partial P}{\partial X}+\left(\frac{\partial^{2} U}{\partial X^{2}}+\frac{\partial^{2} U}{\partial Z^{2}}\right)\right)=0$

$\frac{\partial^{2} W}{\partial T^{2}}+\left(\frac{t_{\text {int }}}{t_{\text {wave }}}\right)^{2}\left(-\frac{\partial P}{\partial Z}+\left(\frac{\partial^{2} W}{\partial X^{2}}+\frac{\partial^{2} W}{\partial Z^{2}}\right)\right)=0$

where $t_{\text {wave }}=h \sqrt{\frac{\rho}{\bar{\mu}}}$ equals the time for an elastic wave to travel through the thickness, $h$, of the interlayer material of mass density $\rho$. For typical representative values $\left(m \approx 10^{3}, \tilde{\omega} \sim 0-\infty\right.$, $\phi \approx 4-4000, \dot{\Delta} \approx 1 \mathrm{~mm} / \mathrm{s}, \mathcal{G}_{c} \approx 0.04-0.2 \mathrm{~J} / \mathrm{m}^{2}, h \approx 40-400 \mu \mathrm{m}$ and $\left.\rho \approx 1 \mathrm{~kg} / \mathrm{m}^{3}\right),\left(\frac{t_{\text {int }}}{t_{\text {wave }}}\right)^{2}=\frac{4}{3 \phi \dot{\Delta}^{2}} \frac{\mathcal{G}_{c}}{\rho h}\left(1+\frac{\mathrm{m}}{1+\tilde{\omega}^{-1}}\right)>>1$. Thus inertia terms are negligible. However, the inertia term will potentially play a significant role for faster pulling speeds for which $\left(\frac{t_{\text {int }}}{t_{\text {wave }}}\right)^{2}$ is not much greater than 1 .

\section{Appendix B}

See appendix Table B1.

\section{Appendix C}

As suggested by a reviewer we include here the effect of the FE mesh on results of the plane strain problem studied in Section 3. Sensitivities of the load-displacement histories and the spatial variations of the contact opening for three $\mathrm{FE}$ meshes in the interlayer are examined. The FE meshes 1, 2, and 3 had, respectively, 5, 5, and 8 elements through the thickness with element widths of $0.05,0.04$ and $0.03 \mathrm{~mm}$. Results plotted in Fig. C1 and Fig. C2 for $\phi=8.25$ and $\phi=41.25$, respectively, are sensitive to the FE mesh at the larger value of $\phi$. A coarse mesh causes spurious oscillations as can be seen from Fig. C2b. However, discrete Fourier transforms of the computed contact openings for mesh 2 and mesh 3 (Fig. C2a) yield the same dominant frequency $(\approx 4.1 \mathrm{~h}$ ).
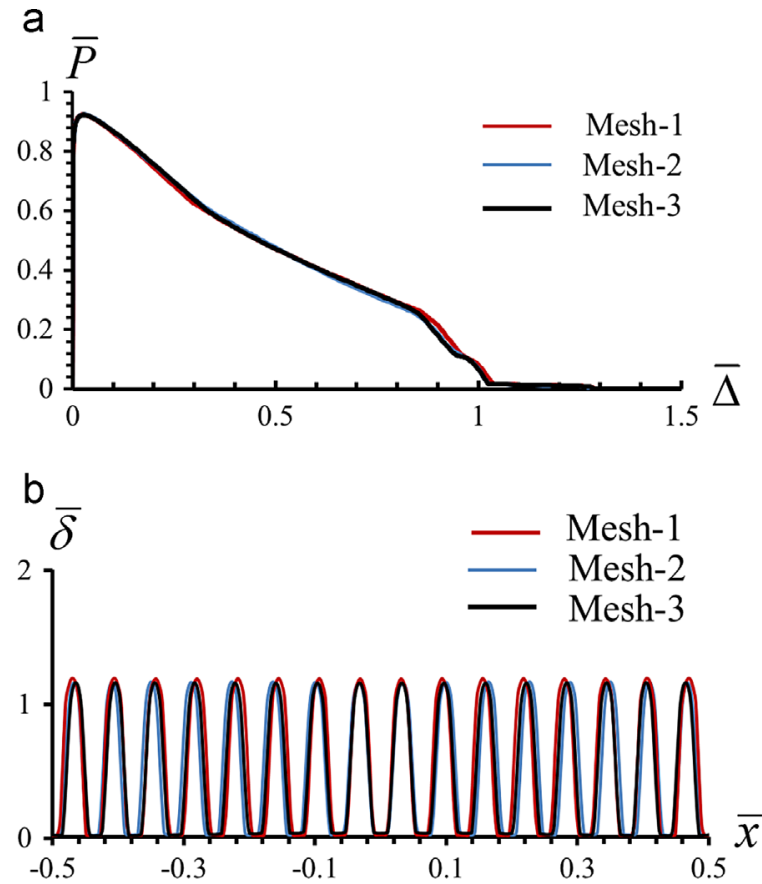

Fig. C1. For $\phi=8.25$, sensitivity to the FE mesh of (a) the dimensionless reaction force $\left(\bar{P}=R / T_{c} \tilde{A}\right)$ vs. the dimensionless displacement, $\bar{\Delta}$, applied at a rate of $1 \mathrm{~mm} / \mathrm{s}$ when $m=3$, and (b) the dimensionless interfacial contact opening when $\Delta=0.54$.
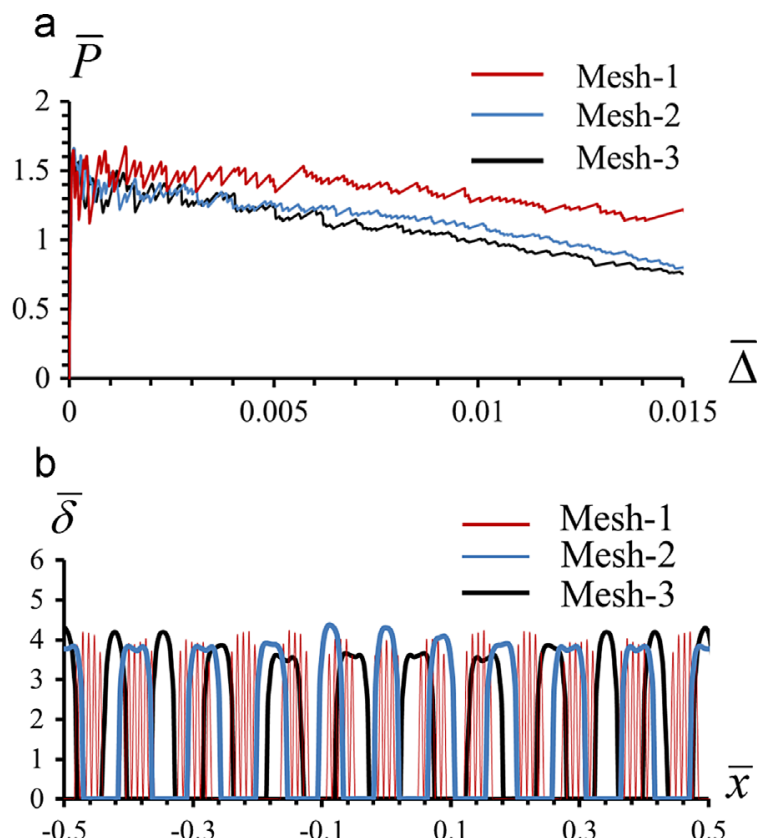

Fig. C2. For $\phi=41.25$, sensitivity to the FE mesh of (a) the dimensionless reaction force vs. dimensionless displacement applied at a rate of $1 \mathrm{~mm} / \mathrm{s}$ when $m=3$, and (b) the dimensionless interfacial contact opening when $\Delta=1.67$.

Table B.1

Values of material parameters in the Prony series at the reference temperature of $45^{\circ} \mathrm{C}$ [45]

\begin{tabular}{|c|c|c|c|c|c|c|c|c|c|c|c|c|c|c|c|c|}
\hline$i$ & 1 & 2 & 3 & 4 & 5 & 6 & 7 & 8 & 9 & 10 & 11 & 12 & 13 & 14 & 15 & 16 \\
\hline$\mu_{i}(\mathrm{MPa})$ & 4.11 & 4.11 & 4.11 & 4.082 & 3.194 & 0.420 & 0.186 & 0.052 & 0.016 & 0.003 & 0.001 & 0.001 & 0.017 & 0.014 & 0.017 & 0.009 \\
\hline$\tau_{i}(\mathrm{~s})$ & $10^{-10}$ & $10^{-9}$ & $10^{-8}$ & $10^{-7}$ & $10^{-6}$ & $10^{-5}$ & $10^{-4}$ & $10^{-3}$ & $10^{-2}$ & $10^{-1}$ & 1 & 10 & $10^{3}$ & $10^{4}$ & $10^{5}$ & $\infty$ \\
\hline
\end{tabular}




\section{References}

[1] Martin WA, Kindt-larsen T, Walker CW. Method and apparatus for demolding ophthalmic contact lenses. US Patent 5, 935; 1999. p. 492.

[2] Mönch W, Herminghaus S. Elastic instability of rubber films between solid bodies. EPL (Eur Lett) 2001:53(4):525.

[3] Chou SY, Zhuang L. Lithographically induced self-assembly of periodic polymer micropillar arrays. J Vac Sci Technol B 1999;17(6):3197-202.

[4] Ghatak, Animangsu Chaudhury, Manoj K Shenoy, Vijay Sharma, Ashutosh. Meniscus instability in a thin elastic film. Phys Rev Lett 2000;85(20):4329.

[5] Ghatak A, et al. Peeling from a biomimetically patterned thin elastic film. In: Proceedings of the royal society of london on mathematical, physical and engineering sciences series A 460; 2004. p. 2725-35.

[6] Nase J, Lindner A, Creton C. Pattern formation during deformation of a confined viscoelastic layer: From a viscous liquid to a soft elastic solid. Phys Rev Lett 2008;101(7):074503.

[7] Lakrout H, Sergot P, Creton C. Direct observation of cavitation and fibrillation in a probe tack experiment on model acrylic pressure-sensitive-adhesives. J Adhes 1999;69(3-4):307-59.

[8] Creton C, Lakrout H. Micromechanics of flat-probe adhesion tests of soft viscoelastic polymer films. J Polym Sci Part B: Polym Phys 2000;38(7):965-79.

[9] Ghatak A, Chaudhury MK. Adhesion-induced instability patterns in thin confined elastic film. Langmuir 2003;19(7):2621-31.

[10] Shenoy V, Sharma A. Pattern formation in a thin solid film with interactions. Phys Rev Lett 2001;86(1):119.

[11] Ghatak A. Confinement-induced instability of thin elastic film. Phys Rev E 2006;73(4):041601.

[12] Adda-Bedia L. Mahadevan. Crack-front instability in a confined elastic film. In: Proceedings of the royal society a: mathematical physical and engineering science 462 ; 2006. p. 3233-51.

[13] Vilmin T, Ziebert F, Raphaël E. Simple view on fingering instability of debonding soft elastic adhesives. Langmuir 2009;26(5):3257-60.

[14] Huang, Shi-Qing Li, Qun-Yang Feng, Xi-Qiao Yu, Shou-Wen. Pattern instability of a soft elastic thin film under van der Waals forces. Mech Mater 2006;38 (1):88-99.

[15] Arun, Narasimhan Sharma, Ashutosh Shenoy, Vijay B Narayan, KS, Electricfield-controlled surface instabilities in soft elastic films, Adv Mater 18 (5), 2006,660-663.

[16] Dugdale D. Yielding of steel sheets containing slits. J Mech Phys Solids 1960;8 (2):100-4.

[17] Barenblatt GI. The mathematical theory of equilibrium cracks in brittle fracture. Adv Appl Mech 1962;7(1):55-129.

[18] Hui CY, Ruina A, Long R, Jagota A. Cohesive zone models and fracture. J Adhes 2011;87(1):1-52.

[19] Hillerborg A, Modéer M, Petersson P-E. Analysis of crack formation and crack growth in concrete by means of fracture mechanics and finite elements. Cem Concr Res 1976;6(6):773-81.

[20] Song SH, Paulino GH, Buttlar WG. A bilinear cohesive zone model tailored for fracture of asphalt concrete considering viscoelastic bulk material. Eng Fract Mech 2006;73(18):2829-48.

[21] Rahul-Kumar P, Jagota A, Bennison SJ, Saigal S, Muralidhar S. Polymer interfacial fracture simulations using cohesive elements. Acta Mater 1999;47 (15):4161-9.

[22] Li S, Thouless MD, Waas AM, Schroeder JA, Zavattieri PD. Use of mode-1 cohesive-zone models to describe the fracture of an adhesively-bonded polymer-matrix composite. Compos Sci Technol 2005;65(2):281-93.

[23] Xu X-P, Needleman A. Numerical simulations of dynamic crack growth along an interface. Int J Fract 1995;74(4):289-324.
[24] Sarkar J, Sharma A, Shenoy V. Adhesion and debonding of soft elastic films: Crack patterns, metastable pathways, and forces. Langmuir 2005;21(4):145769.

[25] Yamaguchi T, Doi M. Debonding dynamics of pressure-sensitive adhesives: 3D block model. Eur Phys J E: Soft Matter Biol Phys 2006;21(4):331-9.

[26] Lindsey, G., et al., The triaxial tension failure of viscoelastic materials. 1963. DTIC Document.

[27] Geubelle PH, Baylor JS. Impact-induced delamination of composites: a 2D simulation. Compos Part B: Eng 1998;29(5):589-602.

[28] Kaelble D. Peel adhesion: micro-fracture mechanics of interfacial unbonding of polymers. Trans Soc Rheol (1957-1977) 1965;9(2):135-63.

[29] Ghatak A, Mahadevan L, Chaudhury MK. Measuring the work of adhesion between a soft confined film and a flexible plate. Langmuir 2005;21(4):127781.

[30] Turon, Albert Davila, Carlos G Camanho, Pedro Ponces Costa J. An engineering solution for mesh size effects in the simulation of delamination using cohesive zone models, Eng Fract Mech 74 (10), 2007, 1665-1682.

[31] Shen B, Paulino G. Direct extraction of cohesive fracture properties from digital image correlation: a hybrid inverse technique. Exp Mech 2011;51 (2):143-63.

[32] Gowrishankar, Shravan Mei, Haixia Liechti, Kenneth M Huang, Rui, A comparison of direct and iterative methods for determining traction-separation relations, International journal of fractureInt J Fract 177177 (2), 2012, 109-128.

[33] Brinson HF, Brinson LC. Polymer engineering science and viscoelasticity: an introduction. Springer; 2007.

[34] Wright T, Ockendon H. A scaling law for the effect of inertia on the formation of adiabatic shear bands. Int J Plast 1996;12(7):927-34.

[35] Lakrout, Hamed Creton, Costantino Ahn, Dongchan Shull, Kenneth R, Influence of molecular features on the tackiness of acrylic polymer melts, Macromolecules 34 (21), 2001, 7448-7458.

[36] Hibbitt, K. and Sorensen, ABAQUS/CAE User's Manual. Hibbitt, Karlsson \& Sorensen, Incorporated; 2012.

[37] Dundurs J. Discussion: Edge-bonded dissimilar orthogonal elastic wedges under normal and shear loading. J Appl Mech 1969;36(3):650-2 (Bogy, DB, 1968, ASME J. Appl. Mech., 35, pp. 460-466).

[38] Bogy DB. Edge-bonded dissimilar orthogonal elastic wedges under normal and shear loading. J Appl Mech 1968;35(3):460-6.

[39] Qian Z, Akisanya A. Wedge corner stress behaviour of bonded dissimilar materials. Theor Appl Fract Mech 1999;32(3):209-22.

[40] Chadegani A, Batra RC. Analysis of adhesive-bonded single-lap joint with an interfacial crack and a void. Int J Adhes Adhes 2011;31(6):455-65.

[41] Wolfram Research I. Mathematica. Wolfram Research, Inc.: Champaign, Illinois; 2014.

[42] Gent A. Cavitation in rubber: a cautionary tale. Rubber Chem Technol 1990;63 (3):49-53.

[43] Murray KV. Characterization of the interfacial fracture of solvated semiinterpenetrating polymer network (s-ipn) silicone hydrogels with a cycloolefin polymer (COP). Virginia Polytechnic Institute and State University; 2011.

[44] Simo J. On a fully three-dimensional finite-strain viscoelastic damage model: formulation and computational aspects. Comput Methods Appl Mech Eng 1987;60(2):153-73.

[45] Tizard III GA. Characterization of the Viscoelastic Fracture of Solvated SemiInterpenetrating Polymer Network (S-IPN) Silicone Hydrogels. Virginia Polytechnic Institute and State University; 2010.

[46] Dillard D. Bending of plates on thin elastomeric foundations. J Appl Mech 1989;56(2):382-6. 